\title{
THE CS MOLECULE IN DIFFUSE INTERSTELLAR CLOUDS
}

\author{
K. DRDLA \\ Department of Chemistry, Princeton University \\ G. R. KNAPP \\ Princeton University Observatory; and AT\&T Bell Laboratories \\ AND \\ E. F. VAN DishoECK \\ Princeton University Observatory; and Division of Geological and Planetary Sciences, California Institute of Technology \\ Received 1988 December 30; accepted 1989 April 20
}

\begin{abstract}
The CS $J=2-1$ emission line at $98 \mathrm{GHz}$ has been searched for in 10 diffuse molecular clouds using the AT\&T Bell Labs $7 \mathrm{~m}$ telescope. Lines are detected at the level $T_{\boldsymbol{A}}^{*} \approx 0.02-0.10 \mathrm{~K}$ from the diffuse cloud toward $o$ Per, the translucent cloud toward HD 169454, the high-latitude cloud toward HD 210121, and from the small cloud $\alpha$ Ori 4. A very tentative detection is reported for the line of sight toward $\zeta$ Oph. CS column densities have been derived by performing statistical equilibrium calculations for the rotational population distribution which included not only collisional excitation by neutral species, but also by electrons. Because CS has a large dipole moment, the electron collisions completely dominate the CS excitation in diffuse clouds, where the electron fraction is high. This result suggests that in general, molecules with large dipole moments may be relatively more easily detectable in diffuse clouds compared with dense clouds by millimeter emission, owing to the efficient electron excitation. A detailed model of the gas-phase sulfur chemistry in diffuse clouds has been developed to interpret the observations. The calculations use existing models for the physical characteristics of the cloud and for the abundances of nonsulfur containing molecules. The predicted CS column densities are affected by uncertainties in the rate at which the molecule photodissociates and in the rate coefficient for the reaction between $\mathrm{S}^{+}$and $\mathrm{CH}$ at low temperatures. The CS photodissociation processes are discussed in detail. It is shown that gas-phase steady state models can reproduce the measured CS column densities for most diffuse clouds using reasonable values for the uncertain rates. Predictions for other sulfurbearing molecules are presented as well. Alternative CS formation schemes are discussed and observational tests suggested.
\end{abstract}

Subject headings: interstellar: molecules - molecular processes

\section{INTRODUCTION}

Although more than 70 molecules have been detected in the interstellar medium, most of these have been observed only in dense clouds, where molecules form more easily because of the higher density and the lower ultraviolet flux. In contrast, only a handful of molecules - all of them diatomic - has been found in diffuse molecular clouds, and many species have been searched for unsuccessfully (see van Dishoeck and Black 1988a for a recent overview). The diffuse clouds are thin enough that they can be studied via visible and ultraviolet absorption line spectra against bright background stars, whereas the dense clouds are observed mostly by millimeter emission lines from the constituent molecules. Because of their modest extinctions, $A_{V}^{\text {tot }} \lesssim 1$ mag, photodestruction processes are important throughout a diffuse cloud and they prevent the buildup of larger molecules. The advantage in studying diffuse clouds, however, is that the observed species and the accompanying network of chemical reactions are simple enough to allow detailed models to be developed. In addition, the thicker diffuse clouds can be studied by both optical absorption and millimeter emission line techniques, which provide complementary information about their physical and chemical structure (Crutcher 1985; van Dishoeck and Black 1989).

Sulfur is the only second-row element for which several compounds have been detected in dense clouds. Although the abundances of some sulfur-bearing molecules relative to the equivalent oxygen-bearing ones appear to be close to those expected on the basis of the overall cosmic sulfur to oxygen ratio, the abundances of most sulfur species are quite different. In diffuse clouds, the searches for the simplest sulfurcontaining molecules have until now been unsuccessful. Snow (1975) obtained an upper limit of $2 \mathrm{~m} \AA$ on the equivalent width of the SH $G-X(0,0)$ line at $1257 \AA$ toward $o$ Per, corresponding to an upper limit on the SH column density of $1.5 \times 10^{11}$ $f^{-1}$, where the oscillator strength $f$ of the transition is not known. Millar and Hobbs 1988) recently found an upper limit on the $\mathrm{SH}^{+}$column density toward $\zeta \mathrm{Oph}$ of $10^{13} \mathrm{~cm}^{-2}$. Very sensitive searches have been made for the $\mathrm{CS}^{+}$ion in 2 diffuse clouds (Ferlet et al. 1986), but despite earlier suggestions (Ferlet et al. 1983), the ion has not been detected toward $\zeta$ Oph at a level $N\left(\mathrm{CS}^{+}\right) \leq 2 \times 10^{11} \mathrm{~cm}^{-2}$. Lines of CS in the $A-X(0$, $0)$ band at $2577 \AA$ were sought without success by Snow (1976a), and resulted in an upper limit of $10^{13} \mathrm{~cm}^{-2}$ toward $\zeta$ Oph. On the other hand, lines of atomic and ionized sulfur are readily detected, and indicate that in most diffuse clouds, sulfur is not significantly depleted from the gas phase (Morton 1975; Snow 1977). The upper limits on the abundances of CS and possibly $\mathrm{SH}$ compared with those of $\mathrm{CO}$ and $\mathrm{OH}$ are much smaller than would be expected on the basis of the overall gas-phase $[\mathrm{S}] /[\mathrm{O}]$ abundance ratio, and indicate that the sulfur chemistry in diffuse clouds differs significantly from that of oxygen. 
Since no sensitive, high-resolution ultraviolet spectrograph is currently available to improve upon the upper limits obtained with the Copernicus satellite, it may be more fruitful to shift the searches for new molecules in diffuse clouds to other wavelength regions, in particular the millimeter region. The CS molecule has its $J=2-1$ transition at $97.981 \mathrm{GHz}$ $(3.06 \mathrm{~mm})$, and is readily observed in dense clouds. Since a sensitive SIS receiver had just become available at this frequency at the AT\&T Bell Labs Telescope, we undertook a search for the CS $J=2-1$ emission from diffuse clouds.

The properties of the clouds selected for observations are discussed in $\S \mathrm{II}$, together with details of the measurements. The excitation of CS in diffuse clouds is computed in $\S$ III, where it is shown that, unlike the case for dark clouds, the excitation is dominated by collisions with electrons. The chemistry of CS is considered in $\S \mathrm{IV}$, with specific reference to the photodissociation processes of the molecule. The calculated CS column densities are found to agree reasonably well with the (uncertain) values inferred from observations. The abundances of other sulfur-bearing molecules are predicted, which could serve as further tests of the proposed diffuse cloud chemistry.

\section{OBSERVATIONS}

\section{a) Selection of Objects and CO Observations}

Although the diffuse clouds were detected classically via their optical absorption line spectra against bright background stars, a large population of clouds of moderate column density has been discovered more recently by their far-infrared emission as observed by the IRAS satellite (Low et al. 1984) and by low-intensity molecular line emission, almost always the $J=1-0$ transition of ${ }^{12} \mathrm{CO}$ (Blitz, Magnani, and Mundy 1984; Keto and Myers 1986; Knapp and Bowers 1988).

In order to facilitate the analysis of the CS data, we chose for observation 10 diffuse clouds from which $\mathrm{CO} J=1-0$ emission is detected. CO $J=1-0$ observations were made for all of the clouds using the AT\&T Bell Laboratories $7 \mathrm{~m}$ telescope in the winter of 1987-1988. The telescope has a half-power beamwidth of $100^{\prime \prime}$ at the $\mathrm{CO} J=1-0$ frequency of $115.27 \mathrm{GHz}$. The receiver temperature was $\sim 280 \mathrm{~K}$ and the observations were made with a velocity resolution of $0.27 \mathrm{~km} \mathrm{~s}^{-1}$. Since the observations were made by frequency switching, care was taken to perform them at a time of year when the cloud velocity relative to the Earth is large, to avoid confusion with emission from atmospheric $\mathrm{CO}$ (which typically has a brightness temperature of $1 \mathrm{~K}$ ). Further details of the observing procedure are given by Knapp and Bowers (1988).

The clouds observed are (1) the classical diffuse clouds towards $\zeta$ Oph and $o$ Per. The visible and ultraviolet absorption spectra of these clouds have been extensively studied and detailed chemical and physical models have been made (van Dishoeck and Black 1986 [hereafter vDB], 1988b). Both ${ }^{12} \mathrm{CO}$ and ${ }^{13} \mathrm{CO}$ observations were made for $o$ Per. These are shown in Figure 1, and the ${ }^{12} \mathrm{CO}$ line toward $\zeta \mathrm{Oph}$ is given in Figure 2. The $\mathrm{CO}$ profile of $\zeta$ Oph shows two velocity components; more sensitive observations at higher velocity resolution by Langer, Glassgold, and Wilson (1987) show that the emission from this cloud contains several velocity components, each with a different spatial extent. (2) We observed one highlatitude cloud, which was detected by its $I R A S 100 \mu \mathrm{m}$ emission and for which a bright early-type background star has been found: HD 210121 (de Vries and van Dishoeck 1988). $\mathrm{CH}, \mathrm{CH}^{+}$and $\mathrm{CN}$ absorption lines have been measured

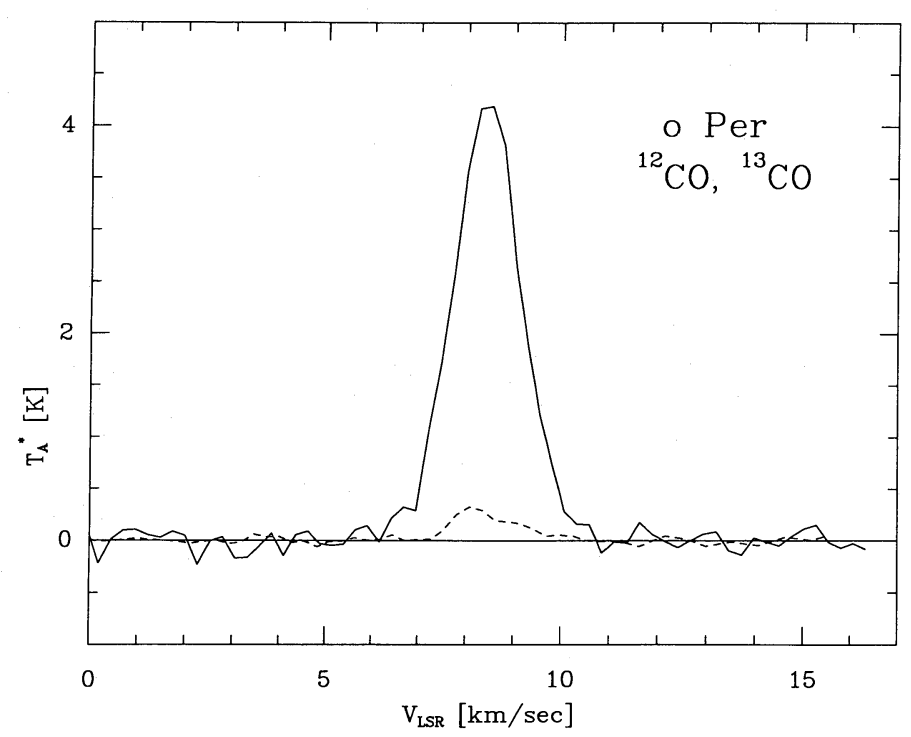

Fig. 1.- ${ }^{12} \mathrm{CO}(J=1 \rightarrow 0)$ (solid line) and ${ }^{13} \mathrm{CO}$ (dashed line) profiles toward $o$ Per.

toward the star. Third, we included one translucent molecular cloud, that toward HD 169454, which has a larger total extinction, $A_{V}^{\text {tot }} \approx 3.3 \mathrm{mag}$. Detailed absorption line observations for this line of sight have been presented by van Dishoeck and Black (1989) and a CO map has been made by Jannuzi et al. (1988). The CO profiles for these clouds are shown in Figures 3 and 4, and the measured $T_{A}^{*}=4.48 \pm 0.16$ toward HD 169454 agrees well with $T_{R}^{*}=4.4-4.7 \mathrm{~K}$ measured by Jannuzi et al. at two different telescopes. The final group of clouds observed are all very small molecular clouds found via their $\mathrm{CO}$ emission. These include four of the five cloudlets toward $\alpha$ Ori (Knapp and Bowers 1988), and two similar objects found at high latitudes: TLC 38 and T Cep. None of these clouds is in front of a bright star, and the only additional information on them comes from CO and (in some cases) far-infrared maps.

For all of the clouds, a single point was observed in CS; for $\zeta$ Oph, $o$ Per, HD 169454, and HD 210121, the direction of the

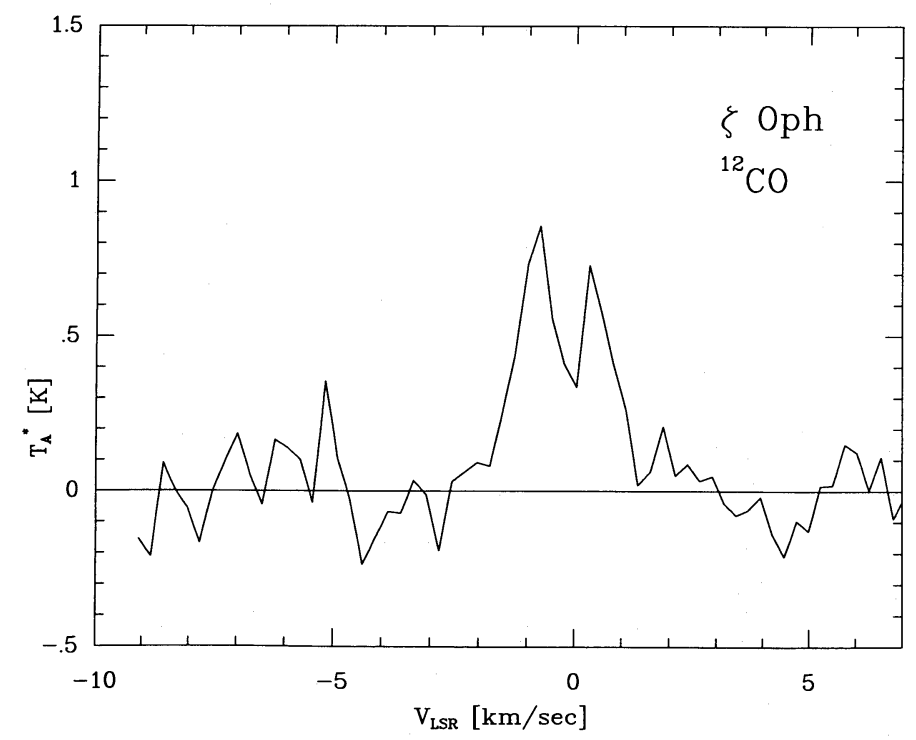

Fig. 2.- $\mathrm{CO}(J=1 \rightarrow 0)$ profile toward $\zeta \mathrm{Oph}$ 


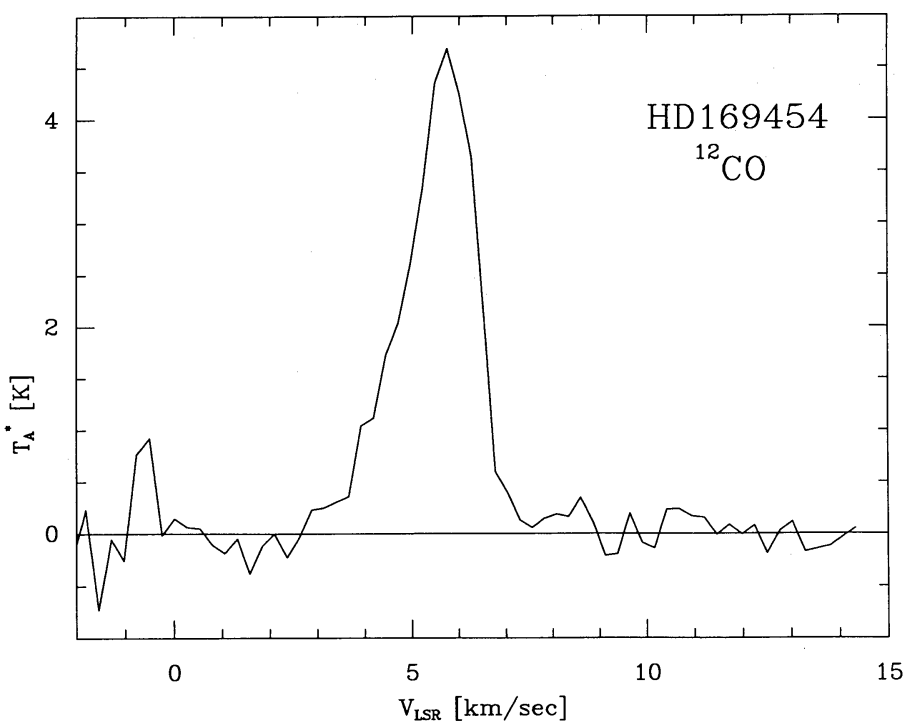

Fig. 3. $-\mathrm{CO}(J=1 \rightarrow 0)$ profile toward HD 169454

star was observed, and for the others, the direction of strongest $\mathrm{CO}$ emission, usually near the cloud center.

\section{b) CS Observations}

The CS observations were made in the winter of 1987-1988 using the $7 \mathrm{~m}$ antenna of AT\&T Bell Laboratories at Holmdel, New Jersey. At the CS $J=2-1$ frequency of $97.98 \mathrm{GHz}$, the telescope half-power beamwidth is $115^{\prime \prime}$ and the main beam efficiency is $\sim 1$. The intensity scale was calibrated by observations of hot (ambient temperature) and cold (liquid $\mathrm{N}_{2}$ ) loads and corrected for atmospheric extinction, and is in units of $T_{A}^{*}$ $(\mathrm{K})$, the Rayleigh-Jeans equivalent brightness temperature observed by a perfect antenna above the atmosphere.

The receiver used a SIS detector cooled to $3 \mathrm{~K}$ by liquid helium. The receiver noise temperature throughout these observations was between 90 and $140 \mathrm{~K}$. The observations were made in clear cold weather with low atmospheric extinction, and the equivalent total system temperature above the atmosphere was less than $250 \mathrm{~K}$.

The back-end filter bank consisted of 128 channels, each of width $0.1 \mathrm{MHz}\left(0.31 \mathrm{~km} \mathrm{~s}^{-1}\right)$. The observations were made by frequency switching, with the expected cloud velocity range in both the signal and reference frequency bands. The individual observations were summed, folded, and a linear or quadratic baseline was removed. The receiver response and atmospheric extinction were measured every half hour or so, and total integration times of $\sim 5-20 \mathrm{hr}$ were accumulated for each cloud.

\section{c) Results}

A single point was observed in each of the 10 clouds. Reliable detections were made from four of the clouds, and a very tentative detection from a fifth $(\zeta \mathrm{Oph})$. For $\zeta \mathrm{Oph}$, the detection was made over only part of the velocity range over which $\mathrm{CO}$ emission is seen (Langer et al. 1987). If confirmed by subsequent observations, this would be the first detection of a new molecule in a classical diffuse cloud since the discovery of absorption lines of $C_{2}$ in 1977 by Souza and Lutz. Figure 5 shows the CS $J=2-1$ line profiles for the five detected clouds and for one undetected cloud ( $\alpha$ Ori 2). The results for all clouds are presented in Table 1 , which lists the cloud name, the

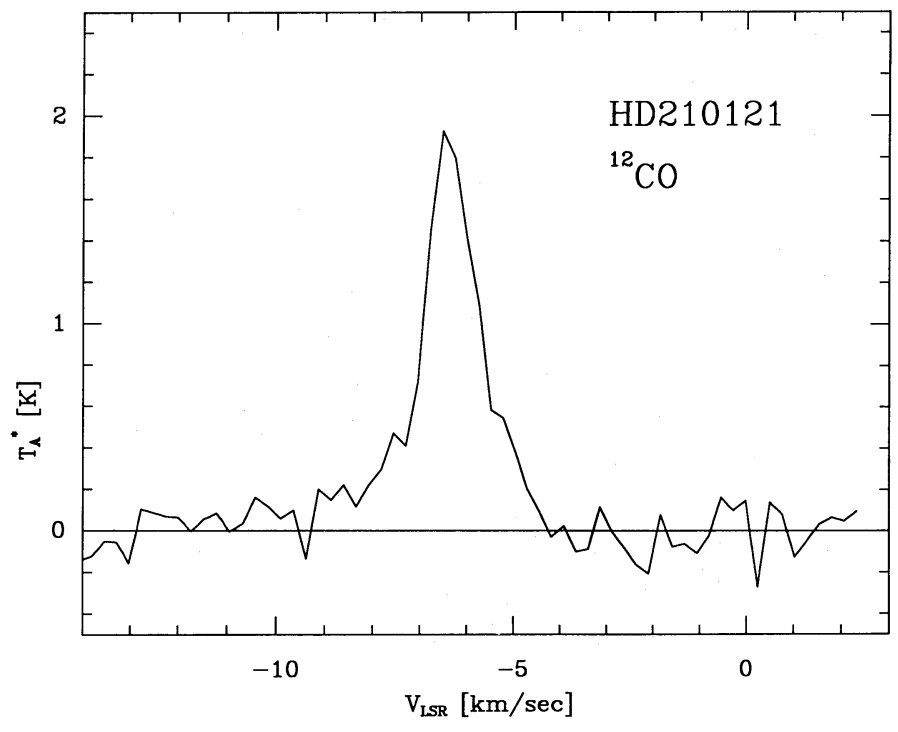

FIG. 4. $-\mathrm{CO}(J=1 \rightarrow 0)$ profile toward HD 210121

observed position and the following parameters for the ${ }^{12} \mathrm{CO}$ and CS observations: the integrated line intensity in $\mathrm{K} \mathrm{km} \mathrm{s}^{-1}$, the peak brightness temperature, the central velocity, and the velocity full width at half-power. The last three quantities are found by Gaussian fitting. For the CS data, the channel-tochannel r.m.s. noise is also listed. The values of the CS integrated intensity were found by summing the CS data over the velocity range containing $\mathrm{CO}$ emission. For $\zeta \mathrm{Oph}$, the data for the two $\mathrm{CO}$ velocity components are listed, and for $o$ Per, the data for both ${ }^{12} \mathrm{CO}$ and ${ }^{13} \mathrm{CO}$ are given.

The results in Figure 5 and Table 1 show that even such a rare molecule as CS is detectable in clouds of quite low density and column density. The agreement between the central velocities and velocity widths of the $\mathrm{CO}$ and $\mathrm{CS}$ lines is excellent, but there appears to be little correlation between their integrated intensities, as illustrated in Figure 6. All of the clouds with $I_{\mathrm{CO}}>3 \mathrm{~K} \mathrm{~km} \mathrm{~s}^{-1}$ are detected in CS, but there is no correspondence between the intensities. This is in contrast with ${ }^{12} \mathrm{CO}$ and ${ }^{13} \mathrm{CO}$, for example, for which the intensities are reasonably well correlated (e.g., Knapp and Bowers 1988). There are thus significant differences in the abundance or excitation, or both, of $\mathrm{CS}$ relative to $\mathrm{CO}$ among the diffuse clouds.

\section{CS ROTATIONAL EXCITATION}

In order to derive total CS column densities from the observation of one line, the rotational population distribution of the molecule needs to be determined. Statistical equilibrium calculations were performed for the lowest 13 levels of the molecule and included the processes of spontaneous emission, stimulated absorption and emission, and collisional excitation and de-excitation. The radiative transfer was treated in terms of mean escape probabilities for an assumed uniform, spherical cloud. The dipole moment used to calculate the rates of the radiative processes was $1.958 \mathrm{D}$ (Winnewisser and Cook 1968), and the $2.7 \mathrm{~K}$ cosmic background field was the only background radiation considered.

The CS excitation thus depends principally on the rates for the collisional processes, determined by the temperature in the cloud and the densities of the various collision partners. At the center of a diffuse cloud, where most of the CS is expected to 

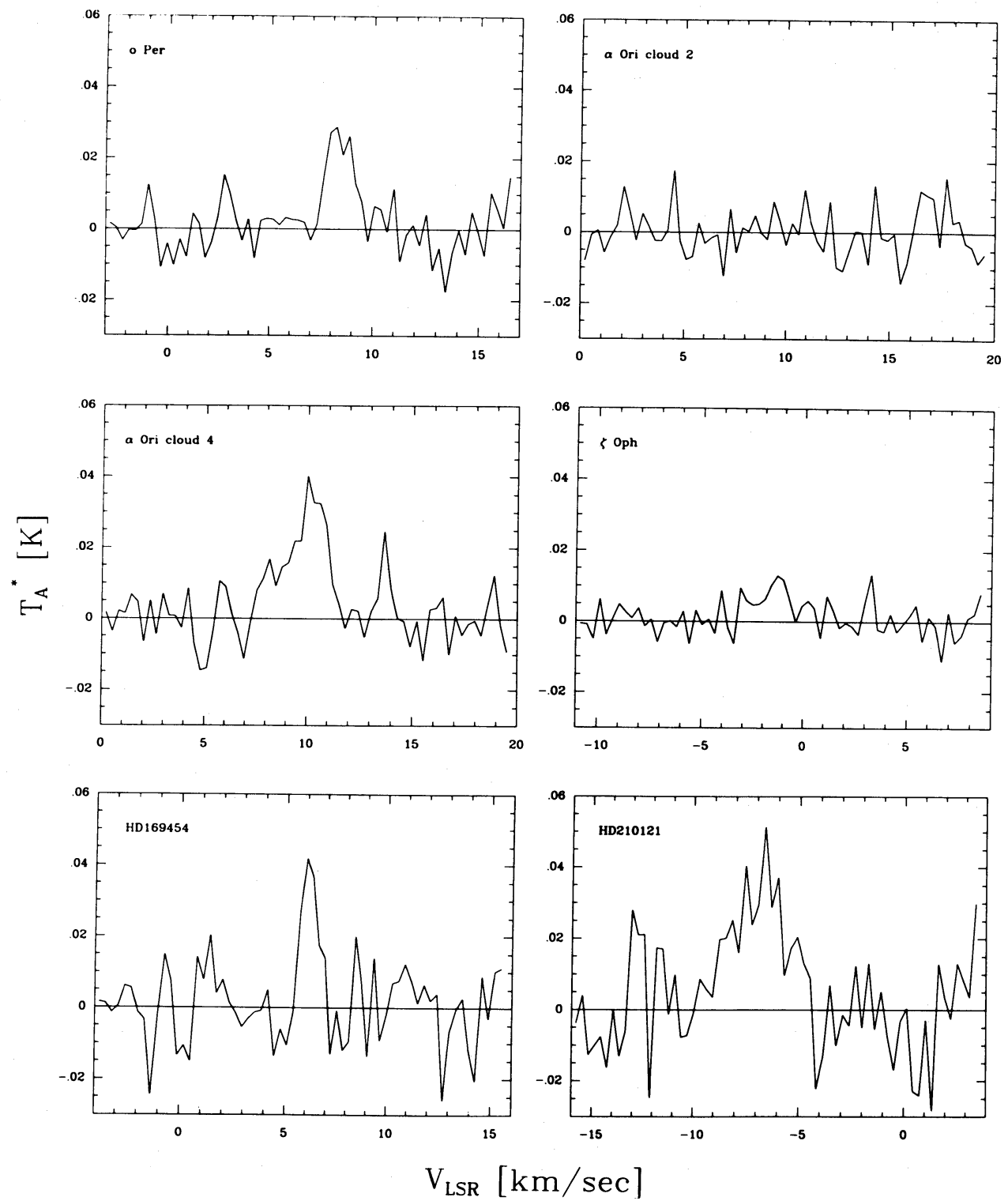

FIG. 5. - CS $(J=2 \rightarrow 1)$ line profiles for the diffuse clouds in the directions of $o$ Per, $\alpha$ Ori (clouds 2 and 4), $\zeta$ Oph, HD 169454, and HD 210121

reside, molecular hydrogen is the most abundant species, although the atomic hydrogen concentration can still be significant (see, e.g., Figure 3 of van Dishoeck and Black 1986). The adopted rate coefficients for collisional (de)excitation of CS by $\mathbf{H}_{2}$ were based on the calculations of Green and Chapman (1978) at $T=20 \mathrm{~K}$. Since collisional excitation by $\mathrm{He}$ is expected to be similar to that by $\mathrm{H}_{2}$, the $\mathrm{He}$ concentration (scaled by the appropriate mass difference) can simply be added to that of $\mathrm{H}_{2}$. Unfortunately, no data are available for $\mathrm{CS}-\mathrm{H}$ collisions. For the case of $\mathrm{CO}$, excitation by $\mathrm{H}$ is intrinsically more efficient than that by $\mathrm{H}_{2}$ (Chu and Dalgarno 1975), and inclusion of $\mathrm{H}$-atom excitation increases the computed $\mathrm{CO}$ brightness temperatures by $\sim 30 \%$ for a cloud like $\zeta$ Oph. In the present calculations for CS, we have not taken excitation by $\mathrm{H}$ explicitly into account, but only implicitly by adding the estimated $\mathrm{H}$ concentration to that of $\mathrm{H}_{2}$. Although the collisional properties for the $\mathrm{CS}-\mathrm{H}$ system are expected to be somewhat different from those for the $\mathrm{CS}_{-} \mathrm{H}_{2}$ system (e.g., $|\Delta J|=1$ transitions will be relatively more important in the former case), this approach is expected to affect the results only slightly.

Much more important is the inclusion of electron excitation into the calculations. This process was considered by Linke and Goldsmith (1980), but subsequently neglected because of the low electron fraction in dense clouds, $x(e)=n(e) / n\left(\mathrm{H}_{2}\right) \lesssim$ $10^{-6}$ (Dalgarno and Lepp 1984). However, in diffuse clouds such as the $\zeta$ Oph cloud, the electron fraction is of order $x(e) \approx 5 \times 10^{-4}$ (van Dishoeck and Black 1986), and even in the centers of thicker translucent clouds, such as that toward HD 169454, the electron fraction is predicted to be $x(e) \approx 5 \times 10^{-5}$ (Jannuzi et al. 1988). For such large electron abundances, electron collisions may well dominate the excitation of a large dipole moment molecule like CS.

No explicit calculations are available for $e$-CS collisional rate coefficients, but a good estimate can be obtained from the formulae of Dickinson et al. (1977). For the case of $e-\mathrm{CN}$ excitation, the rate coefficients obtained in this approximation agree to better than $30 \%$ with those computed explicitly by 
TABLE 1

CO (1-0) ANd CS (2-1) Observations of Diffuse Clouds

\begin{tabular}{|c|c|c|c|c|c|c|c|c|c|c|c|}
\hline Cloud & $\alpha(1950)$ & $\delta(1950)$ & $\begin{array}{c}I_{C O} \\
\left(\mathrm{~K} \mathrm{~km} \mathrm{~s}^{-1}\right)\end{array}$ & $\begin{array}{c}T_{A}^{*}(\mathrm{CO}) \\
(\mathrm{K})\end{array}$ & $\begin{array}{l}V_{c}(\mathrm{CO}) \\
\left(\mathrm{km} \mathrm{s}^{-1}\right)\end{array}$ & $\begin{array}{l}\Delta V(\mathrm{CO}) \\
\left(\mathrm{km} \mathrm{s}^{-1}\right)\end{array}$ & $\begin{array}{c}I_{C S} \\
\left(\mathrm{~K} \mathrm{~km} \mathrm{~s}^{-1}\right)\end{array}$ & $\begin{array}{c}T_{A}^{*}(\mathrm{CS}) \\
(\mathrm{K})\end{array}$ & $\begin{array}{l}V_{c}(\mathrm{CS}) \\
\left(\mathrm{km} \mathrm{s}^{-1}\right)\end{array}$ & $\begin{array}{l}\Delta V(\mathrm{CS}) \\
\left(\mathrm{km} \mathrm{s}^{-1}\right)\end{array}$ & r.m.s. \\
\hline o Per & 034111 & +320754 & $\begin{array}{c}7.5 \pm 0.1 \\
* 0.5 \pm 0.1\end{array}$ & $\begin{array}{l}4.22 \pm 0.06 \\
0.29 \pm 0.02\end{array}$ & $\begin{array}{l}+8.42 \pm 0.01 \\
+8.35 \pm 0.04\end{array}$ & $\begin{array}{l}1.66 \pm 0.03 \\
1.54 \pm 0.10\end{array}$ & $0.042 \pm 0.007$ & \pm 0.005 & $+8.36 \pm 0$ & 0.28 & 0.007 \\
\hline TLC38 & 054959 & +743023 & $1.7 \pm 0.3$ & $0.55 \pm 0.10$ & $-1.75 \pm 0.31$ & $3.17 \pm 0.82$ & - & - & - & - & 0.004 \\
\hline$\alpha$ Ori\#5 & 055159 & +073843 & $1.0 \pm 0.1$ & $1.05 \pm 0.09$ & $+10.83 \pm 0.04$ & $1.09 \pm 0.10$ & - & - & - & - & 0.008 \\
\hline$\alpha$ Ori\#2 & 055224 & +073958 & $2.3 \pm 0.1$ & $1.56 \pm 0.06$ & $+10.67 \pm 0.02$ & $1.23 \pm 0.06$ & - & - & - & - & 0.007 \\
\hline$\alpha$ Ori\#3 & 055319 & +071958 & $1.9 \pm 0.1$ & $0.86 \pm 0.05$ & $+11.22 \pm 0.06$ & $2.05 \pm 0.15$ & - & - & - & - & 0.009 \\
\hline$\alpha$ Ori\#4 & 055344 & +072358 & $3.4 \pm 0.1$ & $2.39 \pm 0.04$ & $+10.34 \pm 0.01$ & $1.36 \pm 0.03$ & $0.061 \pm 0.006$ & $0.037 \pm 0.004$ & $+10.18 \pm 0.09$ & $1.70 \pm 0.23$ & 0.006 \\
\hline$\zeta \mathrm{Oph}$ & 163424 & -102758 & $\begin{array}{l}1.0 \pm 0.2 \\
0.6 \pm 0.1\end{array}$ & $\begin{array}{l}0.77 \pm 0.09 \\
0.67 \pm 0.10\end{array}$ & $\begin{array}{l}-0.76 \pm 0.07 \\
+0.45 \pm 0.07\end{array}$ & $\begin{array}{l}1.21 \pm 0.18 \\
0.95 \pm 0.20\end{array}$ & $\begin{array}{l}0.020 \pm 0.005 \\
-\end{array}$ & $0.013 \pm 0.003$ & $\begin{array}{l}-1.29 \pm 0.17 \\
-\end{array}$ & $\begin{array}{l}1.22 \pm 0.45 \\
-\end{array}$ & 0.005 \\
\hline HD169454 & 182225 & -140026 & $8.6 \pm 0.4$ & $4.48 \pm 0.16$ & $+5.63 \pm 0.03$ & $1.76 \pm 0.07$ & $0.035 \pm 0.009$ & $0.044 \pm 0.009$ & $+6.12 \pm 0.09$ & $0.86 \pm 0.20$ & 0.011 \\
\hline T Cep & 210643 & +681813 & $0.9 \pm 0.1$ & $0.64 \pm 0.07$ & $+1.54 \pm 0.07$ & $1.24 \pm 0.16$ & - & - & - & - & 0.006 \\
\hline HD210121 & 220536 & -034636 & $3.2 \pm 0.1$ & $1.76 \pm 0.07$ & $-6.34 \pm 0.03$ & $1.53 \pm 0.07$ & $0.109 \pm 0.020$ & $0.036 \pm 0.007$ & $-6.91 \pm 0.26$ & $2.89 \pm 0.61$ & 0.015 \\
\hline
\end{tabular}

${ }^{* 13} \mathrm{CO}$.

Allison and Dalgarno (1971). With a CS dipole moment of $1.958 \mathrm{D}$, we obtain $e$-CS collisional deexcitation rate coefficients from level $J+1$ to $J$ of $\sim 3 \times 10^{-6} \mathrm{~cm}^{3} \mathrm{~s}^{-1}$ at $T=10$ $\mathrm{K}$, decreasing to $\sim 2 \times 10^{-6} \mathrm{~cm}^{3} \mathrm{~s}^{-1}$ at $T=60 \mathrm{~K}$ for low $J$. Since the $\mathrm{CS}-\mathrm{H}_{2}$ collisional deexcitation rate coefficients are typically $\sim(2-3) \times 10^{-11} \mathrm{~cm}^{3} \mathrm{~s}^{-1}$, the electron collisions dominate for $x(e)>10^{-5}$. We included all CS- $e$ collisional rate coefficients with $|\Delta J|=1$ at $T=20 \mathrm{~K}$ into the calculations for the lowest $13 \mathrm{CS}$ levels, but neglected larger $\Delta J$ transitions, because they are much less significant. Although all rate coefficients show a weak temperature dependence, we have not taken this into account.

Figure 7 shows the computed CS $J=2-1$ peak RayleighJeans antenna temperatures $T_{A}$ as functions of total CS column density (scaled by the line width $\Delta V$ ), cloud density, electron fraction, and cloud temperature. Table 2 lists the required CS column densities to give $T_{A}=0.03 \mathrm{~K}$ for a range of temperatures, densities, and electron fractions. Several conclusions can be drawn from the figures and table. First, it is important

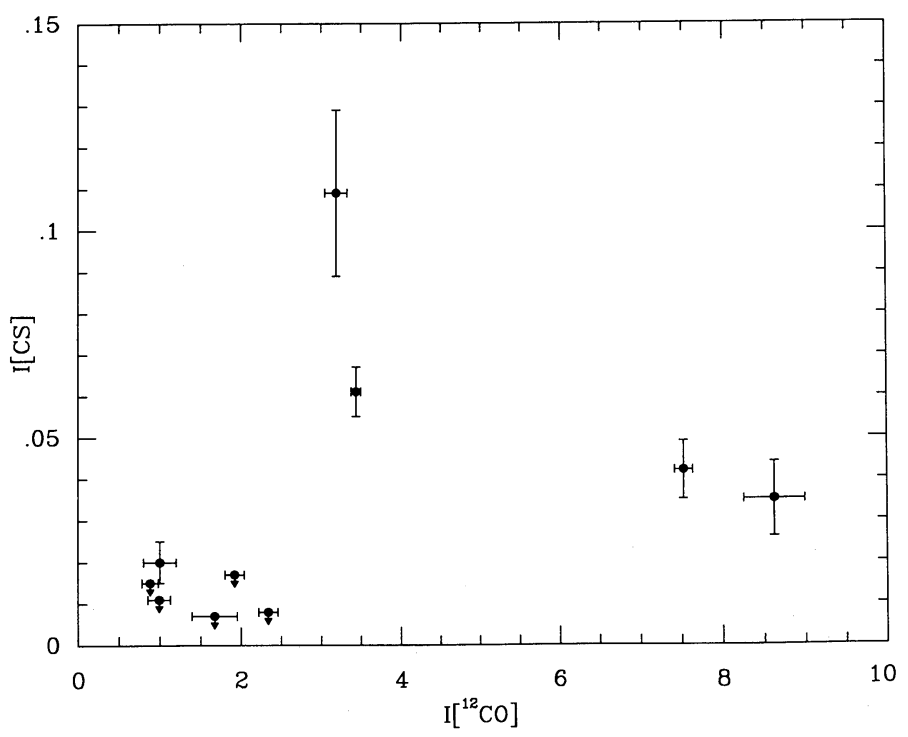

Fig. 6.-Integrated CS $(J=2 \rightarrow 1)$ line intensity vs. integrated CO $(J=1 \rightarrow 0)$ intensity (in $\left.\mathrm{K} \mathrm{km} \mathrm{s}^{-1}\right)$. The $1 \sigma \mathrm{CS}$ upper limits are indicated by arrows. to note that even without electron impact, low-density clouds are detectable in $\mathrm{CS}$ at a level $T_{A} \leq 0.1 \mathrm{~K}$ for reasonable column densities. CS is thus not only a tracer of high-density gas. Second, it is clear that the inclusion of electron collisions significantly affects the results, and that for a classical diffuse cloud, the neglect of electron excitation would result in an overestimate of either the column density or the density by more than an order of magnitude. This example also shows that, compared with dark clouds, molecules with large dipole moments may be more easily detectable in diffuse clouds, because of the efficient electron excitation. In Table 3, the computed $J=1-0 / J=2-1$ and $J=1-0 / J=3-2$ antenna temperature ratios are presented as functions of the electron fraction for $N(\mathrm{CS})=10^{13} \mathrm{~cm}^{-2}$ and various conditions. Because of the different nature of the CS- $e$ interaction compared with $\mathrm{CS}-\mathrm{H}_{2}$, the line ratios change with increasing electron fraction. This effect is particularly noticable for the $J=1-0 / J=3-2$ ratios. Observations of these lines could serve as a confirmation that electron collisions provide the dominant excitation mechanism. At higher CS column densities, greater than $10^{13} \mathrm{~cm}^{-2}$, the transitions become optically thick, which affects the ratios as well.

In order to derive $\mathrm{CO}$ and $\mathrm{CS}$ column densities from the data presented in Table 1 , it is necessary to have some con-

TABLE 2

CS Column Densities Corresponding to $T_{A}(J=2 \rightarrow 1)=0.03 \mathrm{~K}$

\begin{tabular}{crrrrr}
\hline & & \multicolumn{5}{c}{$e / \mathbf{H}_{2}$} \\
\cline { 3 - 6 }$(\mathrm{K})$ & $n\left(\mathrm{H}_{2}\right)$ & \multicolumn{5}{c}{$5 \mathrm{~cm}^{-3}$} & 0 & $5 \times 10^{-5}$ & $1 \times 10^{-4}$ & $5 \times 10^{-4}$ \\
\hline $15 \ldots \ldots \ldots$ & 200 & $1(15)$ & $1(14)$ & $4(13)$ & $5(12)$ \\
& 500 & $6(13)$ & $1(13)$ & $8(12)$ & $2(12)$ \\
& 1000 & $1(13)$ & $5(12)$ & $4(12)$ & $9(11)$ \\
& 3000 & $3(12)$ & $2(12)$ & $1(12)$ & $4(11)$ \\
$30 \ldots \ldots \ldots$ & 200 & $5(14)$ & $6(13)$ & $2(13)$ & $4(12)$ \\
& 500 & $2(13)$ & $9(12)$ & $6(12)$ & $1(12)$ \\
& 1000 & $7(12)$ & $4(12)$ & $3(12)$ & $7(11)$ \\
& 3000 & $2(12)$ & $1(12)$ & $8(11)$ & $3(11)$ \\
& 200 & $2(14)$ & $3(13)$ & $2(13)$ & $4(12)$ \\
& 500 & $1(13)$ & $7(12)$ & $5(12)$ & $1(12)$ \\
& 1000 & $5(12)$ & $3(12)$ & $2(12)$ & $7(11)$ \\
& 3000 & $2(12)$ & $1(12)$ & $7(11)$ & $3(11)$ \\
\hline
\end{tabular}

NoTE.-In this and subsequent tables the notation $a(b)$ indicates $a \times 10^{b}$. 

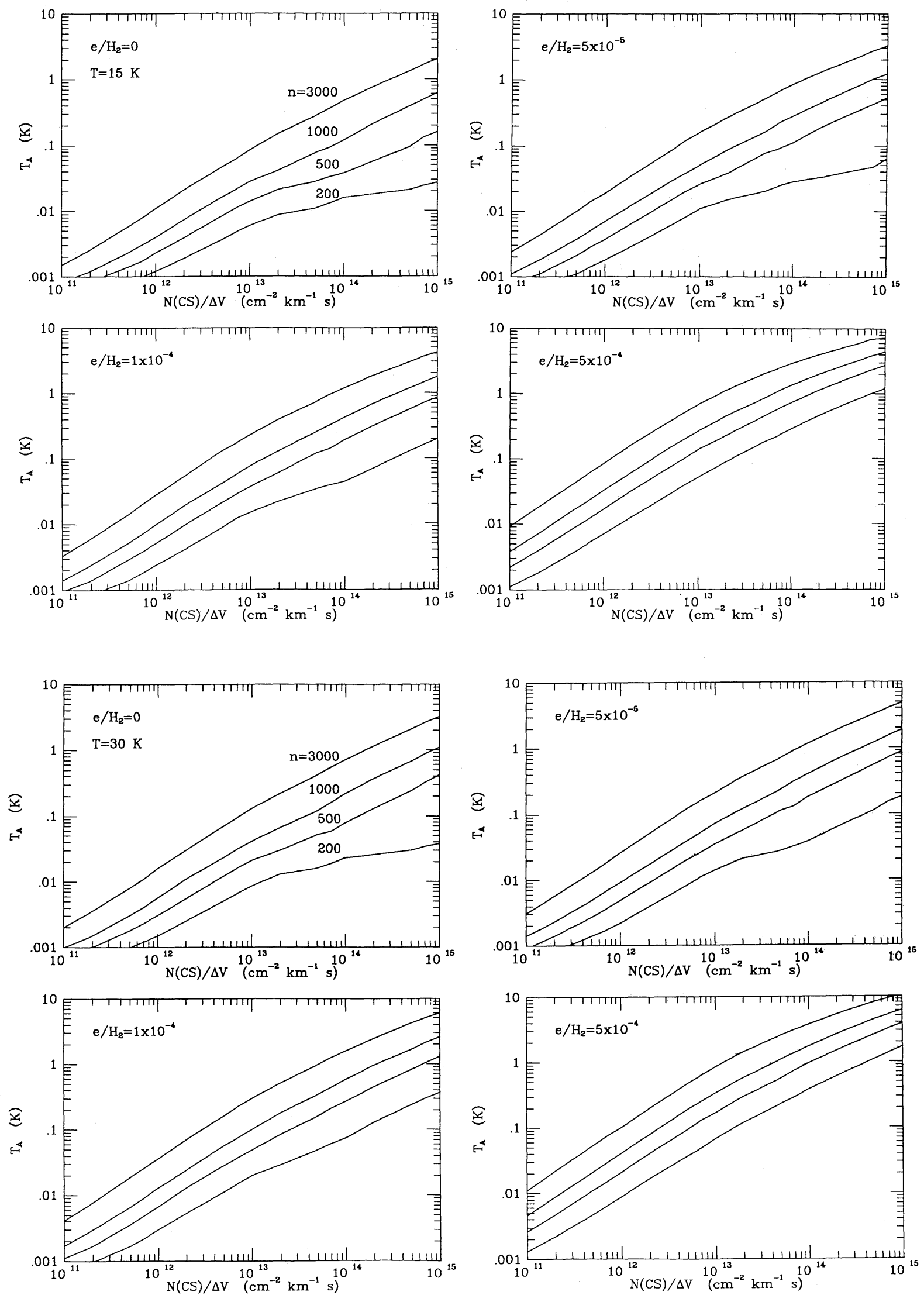

Fig. 7.-Computed CS $(J=2 \rightarrow 1)$ antenna temperatures as functions of CS column density for $T=15,30$, and $50 \mathrm{~K}, n\left(\mathrm{H}_{2}\right)=200,500,1000$, and $3000 \mathrm{~cm}^{-3}$, and electron fractions $e / \mathrm{H}_{2}=0,5 \times 10^{-5}, 1 \times 10^{-4}$ and $5 \times 10^{-4}$. 

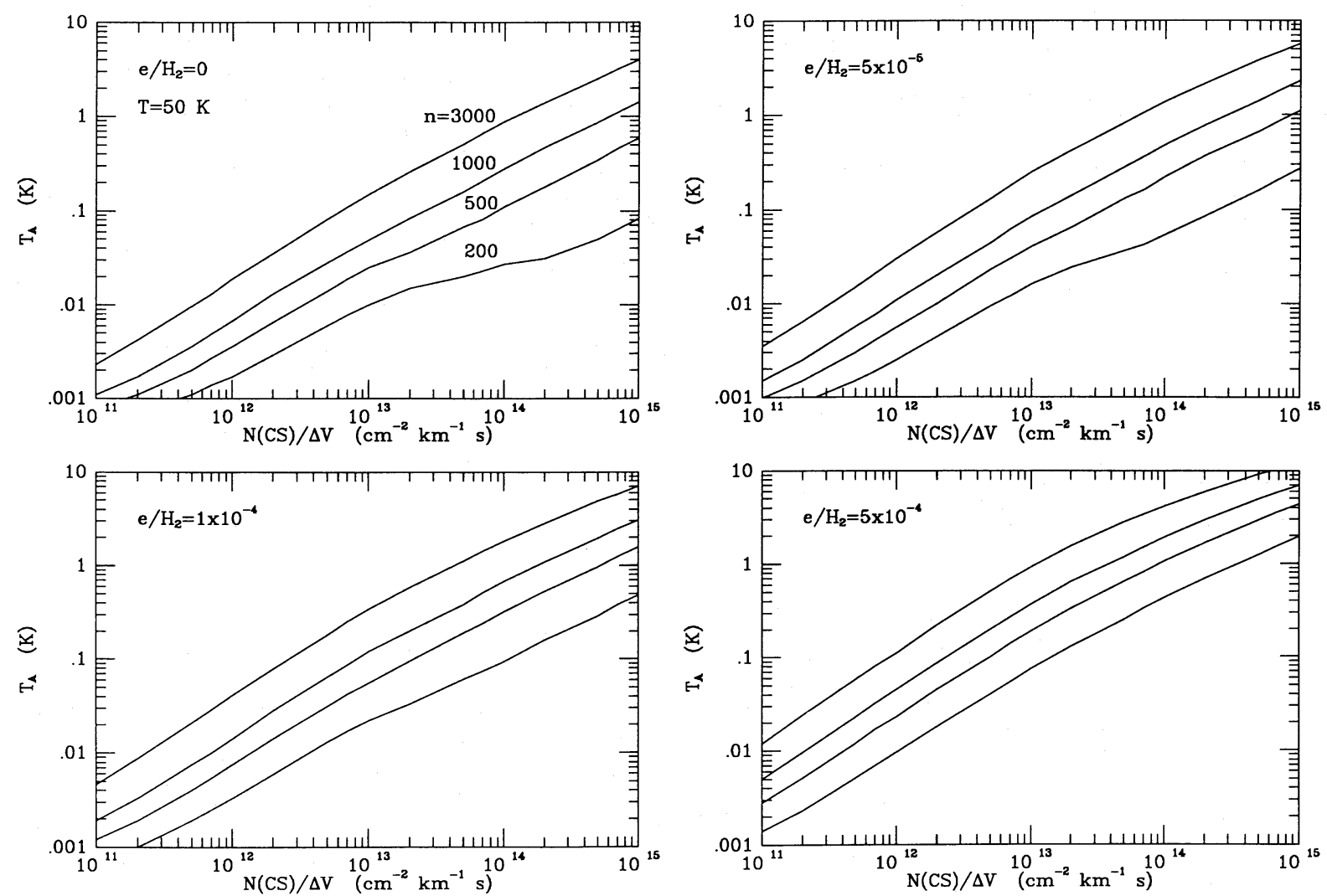

Fig. 7.-Continued

straints on the temperatures and densities in the clouds. For the $\zeta$ Oph and $o$ Per classical diffuse clouds, these parameters can be inferred reasonably well from other diagnostic observations. In particular, the $\mathrm{C}_{2}$ rotational excitation suggests $T \approx 20-30 \mathrm{~K}$ and $n_{\mathrm{H}}=n(\mathrm{H})+2 n\left(\mathrm{H}_{2}\right) \approx 350-800 \mathrm{~cm}^{-3}$ in the center of the $\zeta$ Oph cloud, and somewhat higher temperatures but similar densities in the diffuse cloud in front of $o$ Per. This corresponds to $n\left(\mathrm{H}_{2}\right) \approx 150-400 \mathrm{~cm}^{-3}$ and $n(\mathrm{H}) \approx 50-100$ $\mathrm{cm}^{-3}$. For both clouds, $x(e) \approx 5 \times 10^{-4}$. Note, however, that the analysis of the $o$ Per results is complicated by the fact that a significant part of the $\mathrm{CO}$ emission, and probably also the CS emission, comes from material which lies behind the star (Snow 1975). The density may be higher, and the temperature and

TABLE 3

CS ANTENNA Temperature Ratios for $N(\mathrm{CS})=10^{13} \mathrm{~cm}^{-2}$

\begin{tabular}{|c|c|c|c|c|c|}
\hline \multirow[b]{2}{*}{$T(\mathrm{~K})$} & \multirow[b]{2}{*}{$e / \mathbf{H}_{2}$} & \multicolumn{2}{|c|}{$J=1-0 / 2-1$} & \multicolumn{2}{|c|}{$J=1-0 / 3-2$} \\
\hline & & $n=200$ & 1000 & 200 & $100 \mathrm{~cm}^{-3}$ \\
\hline \multirow[t]{4}{*}{$15 \ldots \ldots \ldots$} & 0 & 2.5 & 2.5 & 7.6 & 7.9 \\
\hline & $5 \times 10^{-5}$ & 3.4 & 3.2 & 14.2 & 13.7 \\
\hline & $1 \times 10^{-4}$ & 3.7 & 3.3 & 18.1 & 16.4 \\
\hline & $5 \times 10^{-4}$ & 3.8 & 2.5 & 24.9 & 14.1 \\
\hline \multirow[t]{4}{*}{$30 \ldots \ldots \ldots$} & 0 & 2.3 & 2.3 & 6.4 & 6.4 \\
\hline & $5 \times 10^{-5}$ & 3.1 & 2.9 & 11.4 & 10.8 \\
\hline & $1 \times 10^{-4}$ & 3.4 & 3.0 & 14.6 & 12.8 \\
\hline & $5 \times 10^{-4}$ & 3.5 & 2.3 & 21.1 & 11.3 \\
\hline \multirow[t]{4}{*}{$50 \ldots \ldots \ldots$} & 0 & 2.3 & 2.2 & 5.9 & 5.8 \\
\hline & $5 \times 10^{-5}$ & 3.0 & 2.7 & 9.5 & 10.2 \\
\hline & $1 \times 10^{-4}$ & 3.3 & 2.8 & 13.0 & 11.3 \\
\hline & $5 \times 10^{-4}$ & 3.4 & 2.2 & 19.3 & 10.2 \\
\hline
\end{tabular}

electron fraction lower in the part of the cloud behind $o$ Per. The temperature in the center of the HD 169454 cloud is low, $T \approx 15 \mathrm{~K}$, and the density somewhat higher compared with classical diffuse clouds, $n_{\mathrm{H}} \approx 600-1500 \mathrm{~cm}^{-1}$, based on the observed $\mathrm{C}_{2}$ excitation (van Dishoeck and Black 1989; Gredel and Münch 1986). The atomic hydrogen density is negligible inside this cloud, and the electron fraction $x(e) \approx 5 \times 10^{-5}$. Little is known about the physical conditions in high-latitude clouds, since no $\mathrm{C}_{2}$ observations are yet available in these directions. However, a comparison of the strength of the $\mathrm{CO}$ $J=3-2$ emission with that of CO $J=1-0$ suggests that the densities in high-latitude clouds are at least as large as those in translucent clouds, $n_{\mathrm{H}} \gtrsim 1000 \mathrm{~cm}^{-3}$ (van Dishoeck et al. 1989). We adopt $T \approx 15-40 \mathrm{~K}$ and $x(e) \approx 5 \times 10^{-5}-10^{-4}$ based on model calculations for the line of sight toward HD 210121 (de Vries and van Dishoeck 1988). The physical conditions in the remaining clouds, $\alpha$ Ori $2-5$, TLC 38 , and T Cep, are also very uncertain, and a range $T=15-40 \mathrm{~K}, n_{\mathrm{H}} \approx 300-1500 \mathrm{~cm}^{-3}$, and $x(e)=\left(5 \times 10^{-5}\right)-\left(2 \times 10^{-4}\right)$ is considered in the interpretation. This range of densities implies line of sight extents of the clouds varying from 0.05 to 1 pc. For comparison, the measured transverse diameters of these clouds are 0.4-1 pc (Knapp and Bowers 1988).

The resulting ranges of inferred $\mathrm{CO}$ and $\mathrm{CS}$ column densities for the various clouds are listed in Table 4, together with a preferred value based on our best estimates of the physical conditions. The table also includes the range of physical parameters used in the analysis. The range of CS column densities for the $\zeta$ Oph cloud is consistent with the upper limit found by Snow (1976a) from absorption line observations. The inferred CS column densities could be tested by searching for 
TABLE 4

CS AND CO COLumn Densities INFERRED From OBSERVATIONS ${ }^{\mathrm{a}}$

\begin{tabular}{|c|c|c|c|c|c|c|c|}
\hline Cloud & $\begin{array}{c}T \\
(\mathrm{~K})\end{array}$ & $\begin{array}{l}n\left(\mathbf{H}_{2}\right)^{\mathrm{b}} \\
\left(\mathrm{cm}^{-3}\right)\end{array}$ & $n(e) / n\left(\mathbf{H}_{2}\right)$ & $\begin{array}{l}N(\mathrm{CS}) \\
\left(\mathrm{cm}^{-2}\right)\end{array}$ & $\begin{array}{l}N(\mathrm{CS})_{\mathrm{pr}}^{\mathrm{c}} \\
\left(\mathrm{cm}^{-2}\right)\end{array}$ & $\begin{array}{l}N(\mathrm{CO}) \\
\left(\mathrm{cm}^{-2}\right)\end{array}$ & $\begin{array}{c}N(\mathrm{CO})_{\mathrm{pr}}^{\mathrm{c}} \\
\left(\mathrm{cm}^{-2}\right)\end{array}$ \\
\hline$o$ Per & $30-50$ & $200-700$ & $5(-5)-2(-4)$ & $4(12)-8(13)$ & $1(13)$ & $(0.7-3)(16)$ & $2(16)$ \\
\hline TLC $38 \ldots \ldots \ldots$ & $20-40$ & $200-700$ & $5(-5)-2(-4)$ & $\leq 2(13)$ & $\ldots$ & $(1-4)(15)$ & $2(15)$ \\
\hline$\alpha$ Ori $5 \ldots \ldots \ldots$ & $20-40$ & $200-700$ & $5(-5)-2(-4)$ & $\leq 2(13)$ & $\ldots$ & $(0.8-3)(15)$ & $2(15)$ \\
\hline$\alpha$ Ori $2 \ldots \ldots \ldots \ldots$ & $20-40$ & $200-700$ & $5(-5)-2(-4)$ & $\leq 2(13)$ & .. & $(2-5)(15)$ & $3(15)$ \\
\hline$\alpha$ Ori 3 & $20-40$ & $200-700$ & $5(-5)-2(-4)$ & $\leq 5(13)$ & & $(1-4)(15)$ & $3(15)$ \\
\hline$\alpha$ Ori 4 & $15-40$ & $200-700$ & $5(-5)-1(-4)$ & $1(13)-1(14)$ & $2(13)$ & $(3-15)(15)$ & $5(15)$ \\
\hline$\zeta \mathrm{Oph}^{\mathrm{d}}$ & $20-30$ & $200-500$ & $2(-4)-5(-4)$ & $7(11)-5(12)$ & $2(12)$ & $(1-3)(15)$ & $2(15)$ \\
\hline HD 169454 & $15-30$ & $300-1000$ & $5(-5)-1(-4)$ & $4(12)-7(13)$ & $3(13)$ & $(1-5)(16)$ & $3(16)$ \\
\hline T Cep ....... & $20-40$ & $200-700$ & $5(-5)-2(-4)$ & $\leq 2(13)$ & & $(0.5-2)(15)$ & $1(15)$ \\
\hline HD 210121 & $15-40$ & $300-1000$ & $5(-5)-1(-4)$ & $4(12)-1(14)$ & 2(13) & $(2-8)(15)$ & $3(15)$ \\
\hline
\end{tabular}

a The table lists the range of column densities consistent with observations and the range of physical parameters adopted in the analysis.

${ }^{b}$ Estimated concentration of $\mathrm{H}$ included; see text.

${ }^{c}$ Preferred value of the column densities.

d Component 1 only.

ultraviolet lines of CS in the $A-X(0,0)$ band at $2577 \AA$ using the high-resolution spectrograph on the Hubble Space Telescope (HST). Predicted equivalent widths for the $R(0)$ line, based on an oscillator strength $f_{00}=0.0096$ for the $A-X(0,0)$ band (Carlson et al. 1979) and assuming that $\sim 40 \%$ of the molecules are in the $J=0$ level, are presented in Table 5 . The $R(1)$ and $Q(1)$ lines arising from the $J=1$ level, which contains $\sim 45 \%$ of the population, are predicted to be about a factor of 2 weaker. The adopted electron fractions could be checked through ultraviolet observations of $\mathrm{C}^{+}$and $\mathrm{C}$ - the principal supplier of electrons.

\section{SULFUR CHEMISTRY}

\section{a) Adopted Gas-Phase Model}

The sulfur chemistry in dense interstellar clouds has been outlined by Oppenheimer and Dalgarno (1974) and Prasad and Huntress $(1980,1982)$, and additional aspects have been discussed by Duley, Millar, and Williams (1980), Millar et al. (1985, 1986), and Pineau des Forêts, Roueff, and Flower $(1986 a)$. The chemical model used in this work incorporated virtually all exothermic gas-phase reactions listed in these references $(\sim 160$ in total) to determine the abundances of 24 sulfur compounds: $\mathrm{S}, \mathrm{S}^{+}, \mathrm{S}^{-}, \mathrm{SH}, \mathrm{H}_{2} \mathrm{~S}, \mathrm{SH}^{+}, \mathrm{H}_{2} \mathrm{~S}^{+}, \mathrm{H}_{3} \mathrm{~S}^{+}, \mathrm{CS}$, $\mathrm{CS}^{+}, \mathrm{HCS}, \mathrm{H}_{2} \mathrm{CS}, \mathrm{HCS}^{+}, \mathrm{H}_{2} \mathrm{CS}^{+}, \mathrm{H}_{3} \mathrm{CS}^{+}, \mathrm{OCS} \mathrm{OCS}^{+}, \mathrm{SO}$, $\mathrm{SO}_{2}, \mathrm{SO}^{+}, \mathrm{HSO}^{+}, \mathrm{NS}, \mathrm{NS}^{+}$, and $\mathrm{HNS}^{+}$. In diffuse clouds, the primary destruction route of the neutral molecules is photodissociation, and the adopted photorates are listed in Table 6 (see also van Dishoeck 1988). Most of them are either ill determined, or pure guesses, and this uncertainty significantly affects the results. Only single exponential decays were considered.
The physical structure of the models was taken from the work of vDB. These models provide the temperature, density and ultraviolet flux at each depth into a (plane-parallel) cloud. They were also the source of the abundances of all non-sulfurcontaining molecules at each depth, which were held fixed in the calculations. The original models of vDB have been updated slightly in this study to incorporate recent developments. These changes include a larger $\mathrm{CO}$ photodissociation rate (van Dishoeck and Black 1988b), larger photoionization rates for $\mathrm{CH}, \mathrm{CH}_{2}$ and $\mathrm{CH}_{3}$, and the addition of metals to the network (including $\mathrm{S}$ and $\mathrm{S}^{+}$) which affects primarily the $\mathrm{C}^{+} / \mathrm{C}$ abundance ratio (Lepp et al. 1988).

The abundance of each of the 24 sulfur species was deter-

TABLE 6

ADOPTED PHOTODESTRUCTION RATES

\begin{tabular}{|c|c|c|c|c|}
\hline Species & Products & $\begin{array}{c}k_{\mathrm{pd}}^{\mathrm{o}} \\
\left(\mathrm{s}^{-1}\right)\end{array}$ & $\gamma^{\mathbf{b}}$ & References \\
\hline $\mathrm{S} \ldots \ldots \ldots \ldots$ & $\mathrm{S}^{+}+e$ & $6(-10)$ & 2.6 & 1 \\
\hline$S^{-} \ldots \ldots \ldots$ & $\mathrm{S}+e$ & $5(-7)$ & 0.9 & 2 \\
\hline SH $\ldots \ldots \ldots \ldots$ & $\mathrm{S}+\mathrm{H}$ & $1(-9)$ & 1.4 & 1 \\
\hline SH ............. & $\mathrm{SH}^{+}+e$ & $6(-10)$ & 2.6 & 5 \\
\hline $\mathrm{H}_{2} \mathrm{~S} \ldots \ldots \ldots$ & $\mathrm{SH}+\mathrm{H}$ & $3(-9)$ & 1.9 & 1 \\
\hline $\mathrm{H}_{2} \mathrm{~S} \ldots \ldots \ldots$ & $\mathrm{H}_{2} \mathrm{~S}^{+}+e$ & $7(-10)$ & 2.7 & 1 \\
\hline $\mathrm{SH}^{+} \ldots \ldots \ldots$ & $\mathrm{S}^{+}+\mathrm{H}$ & $3(-10)$ & 1.8 & 1 \\
\hline $\mathrm{H}_{2} \mathrm{~S}^{+} \ldots \ldots \ldots$ & $\mathrm{SH}^{+}+\mathrm{H}$ & $4(-10)$ & 2.0 & 3 \\
\hline CS $\ldots \ldots \ldots \ldots$ & $S+C$ & $1(-9)$ & 2.0 & 4 \\
\hline CS $\ldots \ldots \ldots \ldots$ & $\mathrm{CS}^{+}+e$ & $2(-10)$ & 3.0 & 5 \\
\hline $\mathrm{CS}^{+} \ldots \ldots \ldots$ & $\mathrm{S}^{+}+\mathrm{C}$ & $2(-10)$ & 2.0 & 3 \\
\hline HCS ......... & $\mathrm{CS}+\mathrm{H}$ & $4(-10)$ & 2.0 & 3 \\
\hline $\mathrm{H}_{2} \mathrm{CS} \ldots \ldots$ & $\mathrm{CS}+\mathrm{H}_{2}$ & $2(-10)$ & 2.0 & 3 \\
\hline $\mathrm{HCS}^{+} \ldots \ldots$ & $\mathrm{CS}^{+}+\mathrm{H}^{+}$ & $4(-10)$ & 2.0 & 3 \\
\hline OCS $\ldots \ldots \ldots$ & $\mathrm{S}+\mathrm{CO}$ & $4(-9)$ & 1.7 & 1 \\
\hline OCS $\ldots \ldots \ldots$ & $\mathrm{OCS}^{+}+e$ & $7(-10)$ & 3.0 & 1 \\
\hline SO $\ldots \ldots \ldots \ldots$ & $\mathrm{S}+\mathrm{O}$ & $4(-9)$ & 2.0 & 1 \\
\hline SO $\ldots \ldots \ldots \ldots$ & $\mathrm{SO}^{+}+e$ & $6(-10)$ & 2.5 & 1 \\
\hline $\mathrm{SO}_{2} \ldots \ldots \ldots$ & $\mathrm{SO}+\mathrm{O}$ & $2(-9)$ & 1.9 & 1 \\
\hline NS ............ & $\mathbf{S}+\mathbf{N}$ & $2(-10)$ & 2.0 & 3 \\
\hline
\end{tabular}

a Photodissociation rate in $\mathrm{s}^{-1}$ in the unattenuated interstellar radiation field of Draine 1978. The rate at the boundary of a finite slab illuminated on both sides is about half the listed rate.

${ }^{b}$ Adopted exponential decay parameter (see van Dishoeck 1988).

REFERENCES. - (1) van Dishoeck 1988; (2) Prasad and Huntress 1980; (3) guess; (4) this paper; (5) Pineau des Forêts, Roueff, and
Flower 1986a. 
mined at each depth into the cloud, based upon the conditions provided by the vDB models. The abundances were then integrated over depth to provide column densities for comparison with observations.

\section{b) Chemistry of CS in Diffuse Clouds}

Although chemically, sulfur is usually considered to be similar to oxygen, the two species behave very differently in diffuse interstellar clouds. This is due mostly to the fact that sulfur, with an ionization potential of $10.4 \mathrm{eV}$, is readily ionized by the interstellar radiation field. Oxygen, on the other hand, has an ionization potential slightly larger than the Lyman limit of $13.6 \mathrm{eV}$, and is thus mostly neutral. However, the formation of sulfur-hydrides is hindered by the fact that in cold clouds, the reactions of $\mathrm{S}^{+}, \mathrm{SH}^{+}$, and $\mathrm{H}_{2} \mathrm{~S}^{+}$with $\mathrm{H}_{2}$ all proceed very slowly, in contrast with the analog reactions for oxygen. The alternative initiating reaction of $\mathrm{S}$ with $\mathrm{H}_{3}^{+}$to form $\mathrm{H}_{2} \mathrm{~S}^{+}$is thought to be very slow as well (Huntress 1977). As a consequence, the predicted amounts of sulfur hydrides in the models are small, and virtually no CS is formed through reactions of $\mathrm{C}^{+}$with, e.g., $\mathrm{SH}$.

Significant amounts of CS can, however, be formed in diffuse clouds through the series of gas-phase reactions

$$
\begin{gathered}
\mathrm{S}^{+}+\mathrm{CH} \rightarrow \mathrm{CS}^{+}+\mathrm{H}, \\
\mathrm{S}^{+}+\mathrm{C}_{2} \rightarrow \mathrm{CS}^{+}+\mathrm{C}, \\
\mathrm{CS}^{+}+\mathrm{H}_{2} \rightarrow \mathrm{HCS}^{+}+\mathrm{H}, \\
\mathrm{HCS}^{+}+e \rightarrow \mathrm{CS}+\mathrm{H} .
\end{gathered}
$$

An additional formation route is through the neutral-neutral reactions of $\mathrm{S}$ with $\mathrm{CH}$ and $\mathrm{C}_{2}$ to form CS directly. It is important to note that $\mathrm{CS}^{+}$is able to react with $\mathrm{H}_{2}$, which allows the chemistry to proceed, although there is some competition from the dissociative recombination of $\mathrm{CS}^{+}$. The dissociative recombination of $\mathrm{HCS}^{+}$with electrons, reaction (4), is unusually slow (Millar et al. 1986), but $\mathrm{HCS}^{+}$is also unreactive with $\mathrm{H}_{2}$ and other abundant interstellar species. Thus essentially all $\mathrm{HCS}^{+}$still leads to CS.

A crucial parameter in the models is the rate coefficient $k_{1}$ for reaction (1). Initially, the rate coefficients for both reactions (1) and (2) were taken from Prasad and Huntress (1980): $k_{1}=$ $6.2 \times 10^{-10} \mathrm{~cm}^{3} \mathrm{~s}^{-1}$ and $k_{2}=8.1 \times 10^{-10} \mathrm{~cm}^{3} \mathrm{~s}^{-1}$. These rate coefficients are based on simple Langevin estimates, since no direct measurements exist. However, reaction (1) involves an ion and a molecule with a permanent dipole moment $\left[\mu_{D}(\mathrm{CH})=1.46 \mathrm{D}\right]$, so that its rate may be significantly enhanced at low temperatures (Su and Bowers 1975; Sakimoto and Takayanagi 1980; Adams, Smith, and Clary 1985). For example, the rate coefficient for the reaction between $\mathrm{C}^{+}$and $\mathrm{H}_{2} \mathrm{O}\left[\mu_{D}\left(\mathrm{H}_{2} \mathrm{O}\right)=1.94 \mathrm{D}\right]$ is found to be $1.2 \times 10^{-8} \mathrm{~cm}^{3} \mathrm{~s}^{-1}$ at $T=27 \mathrm{~K}$ (Marquette et al. 1985; Rowe 1988). If the "locked dipole" formulation as specified by Herbst and Leung (1986) is used, the rate coefficient for the $\mathrm{S}^{+}+\mathrm{CH}$ reaction is found to be as large as $k_{1} \approx 7 \times 10^{-8} T^{-1 / 2} \mathrm{~cm}^{3} \mathrm{~s}^{-1}$. This value is likely to be an upper limit to the rate coefficient. In order to investigate the effect of an enhanced rate for reaction (1), we have performed calculations with a slightly lower value, $k_{1}=2$ $\times 10^{-9}(T / 300)^{-1 / 2} \mathrm{~cm}^{3} \mathrm{~s}^{-1}$.

$\mathrm{CS}$, once formed, can be destroyed by photodissociation, photoionization, charge-transfer reactions, and reactions with $\mathrm{H}_{3}^{+}$. However, the latter three processes are not important destruction mechanisms, since they form either $\mathrm{CS}^{+}$or $\mathrm{HCS}^{+}$, which for the most part lead back to CS. Thus, the dominant destruction route of $\mathrm{CS}$ in diffuse clouds is photodissociation, with destruction by $\mathrm{He}^{+}$only significant in denser clouds. Unfortunately, the photodissociation rate of CS is highly uncertain. Recent laboratory studies of the vacuum ultraviolet absorption spectrum of the isovalent $\mathrm{CO}$ molecule (Letzelter $e t$ al. 1987; Yoshino et al. 1989) have demonstrated that the photodissociation of that molecule proceeds through a series of discrete absorptions into predissociating states. The photodissociation of interstellar $\mathrm{CO}$ is discussed in detail by van Dishoeck and Black (1988b) and Viala et al. (1988). The corresponding spectrum for CS is only partly known. We can, however, make some comparison between the two species. The dissociation energy of CS is $7.35 \mathrm{eV}$, corresponding to a threshold of $1685 \AA$, compared with $11.09 \mathrm{eV}$ or $1118 \AA$ for CO. For the case of $\mathrm{CO}$, the $B^{1} \Sigma^{+}(3 s \sigma)-X^{1} \Sigma^{+}\left(v^{\prime}=0, v^{\prime \prime}=0\right)$ and $(1,0)$ bands lie below the dissociation threshold, but for CS, they lie above it. Indeed, strong predissociations have been observed in the $B-X(0,0)$ band by Stark, Yoshino, and Smith (1987). The theoretical and experimental determinations of the oscillator strengths for the CO $B-X(0,0)$ band range from $f_{00}=(2.3-4.5)$ $\times 10^{-3}$ (Kirby and Cooper 1989; Letzelter et al. 1987). The corresponding value for CS is not known. We assume that the oscillator strength for the CS $B-X$ transition is similar, except for a scaling factor due to the lower excitation energy. If this procedure is applied to the $\operatorname{CO} A^{1} \Pi-X^{1} \Sigma^{+}(0,0)$ band or the $\mathrm{CO}^{+} A^{2} \Pi-X^{2} \Sigma^{+}(0,0)$ band, the resulting oscillator strengths for the corresponding CS transitions agree well with those obtained from measurements (Carlson et al. 1979) or theory (Larsson 1985). Similarly, the $C^{1} \Sigma^{+}(3 p \sigma)-X^{1} \Sigma^{+}(0,0)$ band of $\mathrm{CO}$ lies below the dissociation limit, in contrast with the corresponding band of CS. This CS band also appears diffuse (Donovan, Husain, and Stevenson 1970; Stark, Yoshino, and Smith 1987), indicating that it undergoes rapid predissociation. The CO $C-X(0,0)$ band has a huge oscillator strength, $f_{00} \approx$ 0.06-0.1 (Letzelter et al. 1987; Kirby and Cooper 1989), but no data are available on the corresponding value for CS. We again assume that the oscillator strengths for the two cases are similar, except for an energy scaling factor. Finally, the $E^{1} \Pi(3 p \pi)-X^{1} \Sigma^{+}(0,0)$ band appears to be strongly predissociated in both $\mathrm{CO}$ and $\mathrm{CS}$, and contributes significantly to the photodissociation of both species. The photodissociation of CS through these $B-X, C-X$ and $E-X(0,0)$ bands results in a lower limit to its photodissociation rate in the unattenuated interstellar radiation field as specified by Draine (1978) of $1.5 \times 10^{-10} \mathrm{~s}^{-1}$. The higher lying electronic states of $\mathrm{CO}$ below $13.6 \mathrm{eV}$ are all strongly predissociated with a summed oscillator strength $f \approx 0.3$. It is likely that the equivalent states of CS are also rapidly predissociated, although for CS photoionization can occur as well at $\lambda<1095 \AA$. If a similar effective oscillator strength would apply to CS at $\lambda \approx 1200 \AA$, the photodissociation rate would be increased to $\approx 1 \times 10^{-9} \mathrm{~s}^{-1}$. For comparison, the unshielded $\mathrm{CO}$ photodissociation rate is $2 \times 10^{-10} \mathrm{~s}^{-1}$. In summary, we conclude that the photodissociation rate of interstellar CS is likely to be at least as large as that of $\mathrm{CO}$, and probably even larger, because CS can be photodissociated through more channels at longer wavelengths, where the interstellar radiation field is more intense. We have performed calculations both with a lower limit to the CS photodissociation rate of $2 \times 10^{-10} \mathrm{~s}^{-1}$, and with the larger estimate of $1 \times 10^{-9} \mathrm{~s}^{-1}$. Because of the low abundance of $\mathrm{CS}$ in diffuse clouds, self-shielding does not need to be considered. 
TABLE 7

Computed Column Densities ${ }^{a}$ of CS and Related Species

\begin{tabular}{|c|c|c|c|c|c|c|c|c|c|}
\hline \multirow[b]{2}{*}{ MODEL $^{b}$} & \multirow[b]{2}{*}{$\begin{array}{l}A_{V}^{\text {tot }} \\
\text { (mag) }\end{array}$} & \multirow[b]{2}{*}{$\mathrm{H}_{2}$} & \multirow[b]{2}{*}{$\mathrm{CO}$} & \multirow[b]{2}{*}{$\mathrm{CH}$} & \multirow[b]{2}{*}{$\mathrm{C}_{2}$} & \multicolumn{3}{|c|}{$\begin{array}{c}\text { CS } \\
\text { CALCULATIONS }\end{array}$} & \multirow[b]{2}{*}{$\begin{array}{c}\text { CS } \\
\text { OBSERVED }\end{array}$} \\
\hline & & & & & & $I^{c}$ & $\mathrm{II}^{\mathrm{d}}$ & III $^{\mathbf{e}}$ & \\
\hline $\mathrm{T} 1$ & 0.7 & $5.0(20)$ & $2.5(14)$ & $2.2(13)$ & $2.1(13)$ & $3.3(11)$ & $1.2(12)$ & $4.5(12)$ & $\ldots$ \\
\hline T2 .. & 1.3 & $1.0(21)$ & $2.1(15)$ & $3.5(13)$ & $3.5(13)$ & $8.1(11)$ & $3.2(12)$ & $1.1(13)$ & $\ldots$ \\
\hline T3 ... & 2.0 & $1.5(21)$ & $1.9(16)$ & $6.4(13)$ & $8.5(13)$ & $2.8(12)$ & $1.1(13)$ & $3.5(13)$ & $\ldots$ \\
\hline T4 (HD 169454) & 3.0 & $2.2(21)$ & $7.5(16)$ & $9.4(13)$ & $1.2(14)$ & $5.4(12)$ & $2.3(13)$ & $1.0(14)$ & $4(12)-7(13)$ \\
\hline T5 $\ldots \ldots \ldots \ldots \ldots$ & 3.9 & $3.0(21)$ & $1.4(17)$ & $1.2(14)$ & $1.5(14)$ & $8.6(12)$ & $3.5(13)$ & $1.3(14)$ & $\ldots$ \\
\hline$\zeta$ Per. & 1.0 & $4.8(20)$ & $6.4(13)$ & $2.6(13)$ & $1.2(13)$ & $4.1(10)$ & $1.3(11)$ & $6.8(11)$ & $\ldots$ \\
\hline$\zeta \mathrm{Oph} \ldots \ldots \ldots \ldots \ldots \ldots$ & 0.9 & $4.2(20)$ & $6.2(13)$ & $3.0(13)$ & $1.3(13)$ & $4.3(10)$ & $1.4(11)$ & $6.9(11)$ & $7(11)-5(12):$ \\
\hline$o$ Per $\ldots \ldots \ldots \ldots \ldots \ldots \ldots$ & 1.0 & $4.1(20)$ & $6.1(13)$ & $1.7(13)$ & $8.0(12)$ & $4.0(10)$ & $1.1(11)$ & $7.3(11)$ & $4(12)-8(13)^{g}$ \\
\hline HD $210121 \ldots \ldots \ldots \ldots \ldots$ & 1.0 & $8.0(20)$ & $7.4(15)$ & 4.1(13) & $5.4(13)$ & $1.9(12)$ & $6.8(12)$ & $1.8(13)$ & $4(12)-1(14)$ \\
\hline HD $169454^{\mathrm{h}} \cdots$ & 2.3 & $1.5(21)$ & $2.0(16)$ & $4.7(13)$ & $9.9(13)$ & $1.3(12)$ & $5.2(12)$ & $1.9(13)$ & $4(12)-7(13)$ \\
\hline HD $147889 \ldots \ldots \ldots \ldots$ & 3.0 & $2.0(21)$ & $1.7(16)$ & $7.3(13)$ & $1.6(14)$ & $3.9(12)$ & $1.5(13)$ & 3.1(13) & $\ldots$ \\
\hline
\end{tabular}

a $\mathrm{In}^{-2}$.

b See text for more details of the models; the $\mathrm{CO}, \mathrm{CH}$, and $\mathrm{C}_{2}$ column densities are taken from van Dishoeck and Black 1989.

Calculation I uses $k_{\mathrm{pd}}^{\mathrm{o}}(\mathrm{CS})=10^{-9} \mathrm{~s}^{-1}$ and $k_{1}=6.2 \times 10^{-10} \mathrm{~cm}^{3} \mathrm{~s}^{-1}$.

d Calculation II uses $k_{\mathrm{pd}}^{\mathrm{o}}(\mathrm{CS})=2 \times 10^{-10} \mathrm{~s}^{-1}$ and $k_{1}=6.2 \times 10^{-10} \mathrm{~cm}^{3} \mathrm{~s}^{-1}$

e Calculation III uses $k_{\mathrm{pd}}^{\mathrm{o}}(\mathrm{CS})=2 \times 10^{-10} \mathrm{~s}^{-1}$ and $k_{1}=2 \times 10^{-9}(T / 300)^{-1 / 2} \mathrm{~cm}^{3} \mathrm{~s}^{-1}$.

f Model T4 is equivalent to model 2 presented by Jannuzi et al. 1988.

g Observed value includes contribution from CS located behind the star.

${ }^{\text {h }}$ Model 1 of Jannuzi et al. 1988.

\section{c) Results}

Calculations of the sulfur chemistry have been performed for a series of models. First, the $\zeta$ Oph model G, $o$ Per model B, and $\zeta$ Per model $F$ of vDB have been considered. Second, the sulfur chemistry in the series of translucent cloud models T1-T5 of van Dishoeck and Black $(1988 b, 1989)$ has been computed, to illustrate how the abundances grow with increasing cloud thickness. These models reproduce well the observed trends in the column densities of $\mathrm{CH}$ and $\mathrm{C}_{2}$, the principal precursors of CS. Finally, a number of models for specific clouds, such as the model of de Vries and van Dishoeck (1988) for the HD 210121 cloud, those of Jannuzi et al. (1988) for the HD 169454 cloud, and that of van Dishoeck and Black (1989) for the HD 147889 cloud have been used. In all models, a sulfur depletion factor $\delta_{S} \approx 0.5$ with respect to the solar abundance $[\mathrm{S}] /[\mathrm{H}]=1.7 \times 10^{-5}$ was adopted, based on the results of Lepp et al. (1988) for the $\zeta$ Oph cloud. The adopted carbon depletion factor was $\delta_{C} \approx 0.6$ for the classical diffuse clouds, and $\delta_{C}=0.1$ for the translucent and high-latitude clouds.

The computed column densities for CS are presented in Table 7 and Figure 8, where they are compared with the observational data. For $\mathrm{CS}$, results are given for both the low and the high value of the CS photodissociation rate, and for two values of the rate coefficient for reaction (1). Table 8 gives the results for other sulfur-bearing molecules for the $\zeta$ Oph model, for the HD 210121 model and for model T4, which is appropriate for the HD 169454 cloud. The results for the other sulfurbearing species depend only slightly on the CS
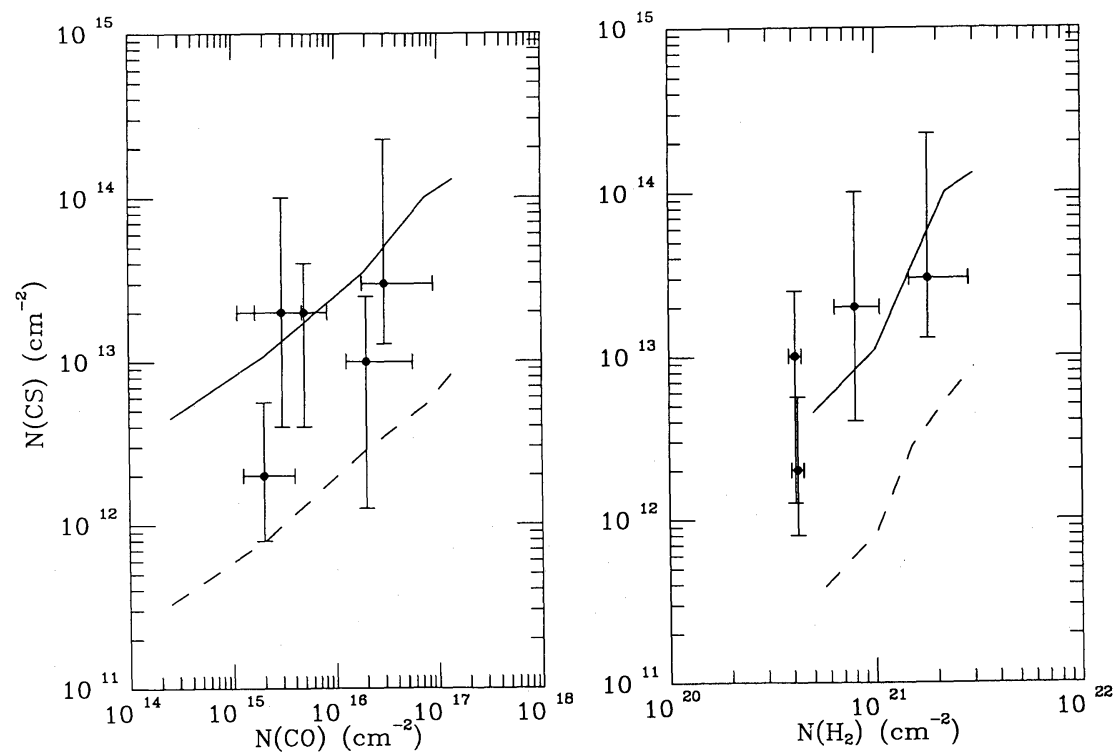

FIG. 8.-Computed CS column densities as functions of computed CO column densities in models T1-T5 for calculation I (dashed line) and calculation III (solid line). The observed values for various clouds are indicated. (b) Same as $(a)$ but for CS column densities as functions of $\mathrm{H}_{2}$ column densities. 
photodissociation rate and are presented for models which adopt the lower value. It appears from Table 8 that CS is indeed the most abundant sulfur-containing molecule in steady state models of diffuse clouds. The computed column densities for $\mathrm{SH}, \mathrm{SH}^{+}$, and $\mathrm{CS}^{+}$are much smaller and are well below the observed upper limits for the $\zeta$ Oph cloud. The comparison between models and observations for $S$ could be improved if charge transfer of $\mathrm{S}^{+}$to large molecules were included in the network (Lepp et al. 1988), but this addition would affect the abundances of other species only slightly. In translucent clouds, $\mathrm{SO}, \mathrm{SO}^{+}$, and $\mathrm{HCS}^{+}$become significant sulfur-bearing molecules. $\mathrm{SO}$ and $\mathrm{SO}^{+}$are formed mostly by reactions of $\mathrm{S}$ and $\mathrm{S}^{+}$with $\mathrm{OH}$.

The calculated CS abundances themselves depend, of course, on the adopted rate coefficients. It appears that the models fall somewhat short of the observed values if the high value for the CS photodissociation rate is used, together with the lower value for the $\mathrm{S}^{+}+\mathrm{CH}$ rate coefficient (calculation I). However, if either the $\mathrm{CS}$ photodissociation rate is lowered, or the $\mathrm{S}^{+}+\mathrm{CH}$ rate enhanced, the model results fall well into the range of inferred column densities for the HD 169454 and HD 210121 clouds. If both rates are taken such as to maximize the CS column density (calculation III), the model results for even the $\zeta$ Oph diffuse cloud approach the (uncertain) measured value. It should be emphasized that the models reproduce (within factors of 2) the observed $\mathrm{CH}, \mathrm{C}_{2}$, and $\mathrm{S}^{+}$column densities wherever available, so that no additional uncertainties are introduced at earlier stages in the network.

The predicted CS column densities also depend on the physical conditions in the models. If all reactions of $\mathrm{S}^{+}$with $\mathrm{CH}$ and $\mathrm{C}_{2}$ eventually lead to $\mathrm{CS}$, the density of $\mathrm{CS}$ can be written as

$$
n(\mathrm{CS})=\frac{k_{1} n\left(\mathrm{~S}^{+}\right) n(\mathrm{CH})+k_{2} n\left(\mathrm{~S}^{+}\right) n\left(\mathrm{C}_{2}\right)}{I_{\mathrm{UV}} k_{\mathrm{pd}}(\mathrm{CS})},
$$

where $n\left(\mathrm{~S}^{+}\right) \approx 1.7 \times 10^{-5} \delta_{S} n_{\mathrm{H}}$. Thus the CS abundance is enhanced by increasing $\delta_{S}$ and $n_{H}$, and by decreasing $I_{\mathrm{Uv}}$. The temperature affects $n(\mathrm{CS})$ through $k_{1}$ : the production of CS is clearly more increased in cold cloud models like that for the HD 169454 cloud, than in warm models, like that for HD 147889 (compare calculations II and III).

Figure 8 shows that, within the large observational errors, the models can reproduce the observed trend in the CS versus CO column density. Part of the scatter in this figure (and that in Fig. 6) may result from the fact that the individual clouds have different physical conditions. In addition, the $\mathrm{CO}$ column densities in some of the clouds (e.g., the HD 169454 and $o$ Per clouds) are large enough for self-shielding to be important (van Dishoeck and Black 1988b). Thus, the CO column densities for these clouds can be significantly enhanced compared with clouds of slightly smaller total thickness, without affecting the column densities of other molecules like CS. In contrast, the ${ }^{12} \mathrm{CO} /{ }^{13} \mathrm{CO}$ column density ratio remains fairly constant (within a factor of 2) over this range of conditions, because the effects of isotope selective photodissociation and temperaturesensitive ion-exchange reactions tend to cancel each other (van Dishoeck and Black 1988b), consistent with observations (Knapp and Bowers 1988).

The comparison between the predicted and observed CS column densities is least favorable for the $\zeta$ Oph cloud. It could simply be that the detection is not real in this direction. Alternatively, the comparison could be enhanced by either increasing the density or decreasing $I_{U V}$ in the models. The appropriate scaling factor $I_{\mathrm{UV}}$ for the interstellar radiation field incident on the $\zeta$ Oph cloud is actually subject to discussion (van Dishoeck and Black 1989; van Dishoeck 1989; Hartquist, Flower, and Pineau des Forêts 1989). In the steady state models, it is constrained principally by the observed high- $J$ population of $\mathrm{H}_{2}$, under the assumption that ultraviolet pumping is the dominant excitation mechanism for these levels. If some other process, such as collisional excitation in shock-heated gas, would play a role as well, the value of $I_{\mathrm{UV}}$ would have been overestimated. Although the magnetohydrodynamic (MHD) shock models have not yet succeeded in reproducing the observed $\mathrm{H}_{2}$ rotational distribution in diffuse clouds without overproducing the abundances of other species (Hartquist et al. 1989), the possibility of a lower value of $I_{\mathrm{UV}}$ needs to be considered in the steady state models. A reduction of $I_{\mathrm{UV}}$ by a factor of $\sim 3$ would improve the agreement between models and observations (compare model T1 with the $\zeta$ Oph model). Such a reduction would help to solve the discrepancies for other molecules like $\mathrm{CN}$ and $\mathrm{CO}$ as well. On the other hand, it would also lead to larger $\mathrm{C}_{2}$ and $\mathrm{CH}$ column densities, so that the rate coefficient for the radiative association reaction between $\mathrm{C}^{+}$and $\mathrm{H}_{2}$ would have to be lowered to $\sim 2 \times 10^{-16}$ $\mathrm{cm}^{3} \mathrm{~s}^{-1}$ in order to not exceed the observed values. The shape of the ultraviolet radiation field at the shortest wavelengths $(\lambda<1000 \AA)$ is also not well known (van Dishoeck and Black $1988 b$ ), but this uncertainty does not affect $\mathrm{CS}, \mathrm{CH}$ or $\mathrm{C}_{2}$, since these molecules photodissociate at longer wavelengths. As discussed by van Dishoeck and Black $(1986,1988 a)$, observations of ultraviolet lines out of vibrationally excited levels of $\mathrm{H}_{2}$ could determine $I_{\mathrm{UV}}$ unambiguously.

\section{d) Alternative CS Formation Schemes}

Can other chemical processes, such as those occurring on grain surfaces or in shock-heated gas, play a role in the formation of sulfur-bearing molecules in diffuse clouds? The production of sulfur-bearing molecules in shock-heated gas has recently been discussed by Pineau des Forêts, Roueff, and

TABLE 8

Predicted Column Densities of Other Sulfur-Bearing Species ${ }^{a}$

\begin{tabular}{|c|c|c|c|c|}
\hline \multirow[b]{2}{*}{ SPECIES } & \multicolumn{2}{|c|}{$\zeta \mathrm{OPH}$} & \multirow[b]{2}{*}{$\begin{array}{c}\text { HD } 210121 \\
\text { Model }\end{array}$} & \multirow[b]{2}{*}{$\begin{array}{c}\text { HD } 169454 \\
\text { Model }\end{array}$} \\
\hline & Model & $\begin{array}{l}\text { OBSERVED } \\
\text { VALUES }^{\text {b }}\end{array}$ & & \\
\hline $\mathrm{S}$ & $2.5(13)$ & $8(13)$ & $5.8(13)$ & $6.1(14)$ \\
\hline $\mathbf{S}^{+}$. & $1.3(16)$ & $7(15)-2(16)$ & $1.0(16)$ & $3.1(16)$ \\
\hline $\mathrm{S}^{-}$ & $2.0(4)$ & $\ldots$ & $9.5(4)$ & $6.1(5)$ \\
\hline SH & $4.2(4)$ & $\leqslant 1(11) f^{-1}$ & $2.1(6)$ & $7.3(7)$ \\
\hline $\mathrm{H}_{2} \mathrm{~S}$ & $4.3(2)$ & $\ldots$ & $6.4(4)$ & $5.4(6)$ \\
\hline $\mathrm{SH}^{+}$ & $4.2(7)$ & $\leqslant 1(13)$ & $4.4(8)$ & $5.7(9)$ \\
\hline $\mathrm{H}_{2} \mathrm{~S}^{+} \ldots \ldots \ldots \ldots$ & $1.3(2)$ & ... & $7.1(4)$ & $2.8(6)$ \\
\hline $\mathrm{CS} \ldots \ldots$ & $6.9(11)$ & $7(11)-5(12):$ & $1.8(13)$ & $1.0(14)$ \\
\hline $\mathrm{CS}^{+} \ldots$ & $6.6(9)$ & $\leqslant 2(11)$ & $1.6(10)$ & $3.1(10)$ \\
\hline HCS $\ldots \ldots \ldots \ldots$ & $1.6(7)$ & ... & $1.0(9)$ & $3.0(10)$ \\
\hline $\mathrm{H}_{2} \mathrm{CS} \ldots \ldots \ldots$ & $4.8(4)$ & $\ldots$ & $7.2(6)$ & $2.6(8)$ \\
\hline $\mathrm{HCS}^{+} \ldots \ldots \ldots$ & $1.7(10)$ & $\ldots$ & $2.1(11)$ & $6.2(11)$ \\
\hline $\mathrm{H}_{2} \mathrm{CS}^{+} \ldots \ldots \ldots$ & $2.8(2)$ & $\ldots$ & $2.6(4)$ & $2.2(5)$ \\
\hline OCS $\ldots \ldots \ldots \ldots$ & $2.4(4)$ & $\ldots$ & $1.3(7)$ & $8.8(9)$ \\
\hline $\mathrm{OCS}^{+} \ldots \ldots \ldots$ & $5.3(7)$ & $\ldots$ & $2.2(9)$ & $9.9(9)$ \\
\hline SO $\ldots \ldots \ldots \ldots$ & $2.5(8)$ & $\ldots$ & 4.8(9) & $1.0(12)$ \\
\hline $\mathrm{SO}_{2} \ldots \ldots \ldots \ldots$ & $2.5(2)$ & $\ldots$ & $5.8(4)$ & $3.3(8)$ \\
\hline $\mathrm{SO}^{+} \ldots \ldots \ldots \ldots$ & $1.5(9)$ & $\cdots$ & $1.8(10)$ & $3.1(11)$ \\
\hline NS $\ldots \ldots \ldots \ldots \ldots$ & $2.5(2)$ & $\ldots$ & $5.6(4)$ & $9.3(6)$ \\
\hline $\mathrm{NS}^{+} \ldots \ldots \ldots \ldots$ & $9.2(5)$ & $\ldots$ & $1.3(7)$ & $1.8(8)$ \\
\hline
\end{tabular}

a The listed values refer to calculation III.

b See introduction for references to the observed values. 
Flower (1986a). Because of the large energy barriers (up to $10,000 \mathrm{~K}$ ) for the important reactions, one-fluid hydrodynamic shocks are not efficient in enhancing the amounts of sulfurhydrides or CS in diffuse clouds for realistic shock speeds. On the other hand, models of MHD shocks produce significant amounts of $\mathrm{SH}^{+}$and a few related species. The MHD shock models that best reproduce the observed $\mathrm{CH}^{+}$column densities in the $\zeta \mathrm{Oph}$ and $o$ Per clouds adopt a magnetic field of 5 $\mu \mathrm{G}$, and shock speeds of 16 and $11 \mathrm{~km} \mathrm{~s}^{-1}$, respectively (Pineau des Forêts et al. 1986b). A low preshock density ratio $n\left(\mathrm{H}_{2}\right) / n(\mathrm{H}) \leq 2$ is needed to avoid overproduction of $\mathrm{CH}$ and $\mathrm{OH}$ compared with observations. For these models, Pineau des Forêts, Roueff, and Flower (1986a) predict CS column densities of $2 \times 10^{12}$ and $3 \times 10^{11} \mathrm{~cm}^{-2}$, respectively, for the two clouds. These CS column densities are slightly larger than those found in the steady state models, and are in the range of the observed values. Note, however, that the shock models use an unattenuated CS photodissociation rate of only $3 \times 10^{-11}$ $\mathrm{s}^{-1}$, nearly an order of magnitude lower than our estimated lower limit of $2 \times 10^{-10} \mathrm{~s}^{-1}$. In addition, the scaling factor $I_{\mathrm{UV}}$ is taken to be 1 , whereas later models (Hartquist et al. 1989) suggest that $I_{\mathrm{UV}} \approx 3-5$ is more realistic. Since photodissociation is the dominant destruction path of CS in shocks, the CS column densities should be lowered by at least one order of magnitude for proper comparison with the steady state models. Thus, the amount of shock-produced CS is at best comparable to that found in steady state. Another argument against a large shock production of CS is the fact that the shock models predict a strong correlation between the amounts of $\mathrm{CH}^{+}$and $\mathrm{CS}$, which is not observed: the measured $\mathrm{CH}^{+}$column density in the HD 210121 cloud, which has the largest CS column density, is significantly less than that found in the $\zeta$ Oph cloud (de Vries and van Dishoeck 1988). Finally, shock-produced CS would be displaced by a few $\mathrm{km} \mathrm{s}^{-1}$ with respect to $\mathrm{CO}$, which is inconsistent with the observations. More sensitive searches for $\mathrm{SH}^{+}$in diffuse clouds (Millar and Hobbs 1988) would be valuable to further constrain the shock models, since this ion can only be produced in significant amounts in shocks.

The grain surface chemistry of sulfur in diffuse clouds has been discussed by Duley et al. (1980). They suggest that the gas-phase $\mathrm{S}^{+}$ions can react with $\mathrm{O}^{-}$or $\mathrm{OH}^{-}$sites at the surfaces of oxide grains to form excited HSO, which subsequently dissociates to form SO. Thus, in clouds in which sulfur is nearly undepleted, SO is predicted to be the most important sulfur-bearing molecule, although its estimated column density, $\approx 10^{11} \mathrm{~cm}^{-2}$, is probably below the current limits of detectability at millimeter wavelengths. Searches for lines of SO in, e.g., the strong $B^{3} \Sigma^{-}-X^{3} \Sigma^{-}$system around $2400 \AA$ toward $\zeta$ Oph and HD 169454 would be worthwhile. CS and SH are only formed efficiently in models in which sulfur is depleted significantly on grains, where it replaces oxygen to form $\mathrm{S}^{-}$and $\mathrm{SH}^{-}$sites. Reactions of $\mathrm{C}^{+}$and $\mathrm{H}^{+}$with these sites then lead to $\mathrm{CS}$ and $\mathrm{SH}$. No detailed calculations have been performed, but the maximum predicted column densities are of the order of $10^{12}-10^{13} \mathrm{~cm}^{-2}$. These predictions will be lowered significantly if the rapid photodissociation of the molecules is taken into account. This CS formation scheme does not appear plausible for the $\zeta$ Oph cloud, where sulfur is nearly undepleted. However, Snow (1976b) has suggested that sulfur is more depleted in the $o$ Per cloud compared with the other diffuse clouds. Our inferred CS column density in that direction is indeed larger than that found toward $\zeta \mathrm{Oph}$, although a large part of the CS detected at millimeter wavelengths may actually be behind the star. More sensitive searches for SH through its absorption lines at $3200 \AA$ toward $o$ Per would be valuable to constrain the grain surface production of $C S$ in that direction.

In summary, it appears that the amount of CS formed in shock-heated gas or through grain surface reactions is at best comparable with that formed in steady state, and probably smaller by at least an order of magnitude.

\section{CONCLUDING REMARKS}

The detection of millimeter emission of CS-albeit very weak - from a number of diffuse molecular clouds indicates that the molecule is more widespread than previously thought, and that it is not only a tracer of the densest parts of interstellar clouds. Although the inferred CS column densities are quite uncertain, it appears that simple gas-phase steady state chemistry can account for the observed abundances, and that no additional shock or grain-surface formation is necessary. The largest uncertainty in the models lies in the photodissociation rate for $\mathrm{CS}$ and the rate coefficient for the $\mathrm{S}^{+}+\mathrm{CH}$ reaction at low temperatures. Theoretical calculations and laboratory experiments similar to those performed recently for $\mathrm{CO}$ should be able to better constrain the CS photodissociation rate. Further experiments on reactions between ions and polar molecules at low temperatures could test the validity of the adopted approximation to the rate coefficient for the $\mathrm{S}^{+}+\mathrm{CH}$ reaction.

An interesting conclusion of this study is that the large dipole moment of CS helps, rather than prevents, the detection of the molecule in diffuse clouds, since electron collisions dominate its excitation. This suggests that searches for other molecules with large dipole moments, such as $\mathrm{HCN}, \mathrm{H}_{2} \mathrm{CO}$, and $\mathrm{C}_{3} \mathrm{H}_{2}$, may also be successful. Because electron collisions favor $|\Delta J|=1$ transitions, observations of the lowest transitions may be most fruitful. For CS, the $J=1 \rightarrow 0$ antenna temperature is predicted to be a factor of $2-4$ higher than that of the $2 \rightarrow 1$ transition. The millimeter observations need to be complemented, however, by absorption line observations, wherever possible. Absorption line observations of, e.g., $\mathrm{C}_{2}, \mathrm{C}$, and $\mathrm{C}^{+}$can constrain the physical parameters such as temperature, density, and electron fraction in the cloud, which are necessary in the analysis of the millimeter data. Future ultraviolet absorption line measurements of CS could better determine the column densities. A fruitful interaction between the two observing techniques is expected in the near future.

The authors are very grateful to J. Bally, A. A. Stark, and R. W. Wilson for the observing time on the Bell Labs $7 \mathrm{~m}$ telescope. The SIS junctions were made by R. E. Miller. They enjoyed many useful discussions with J. H. Black concerning the CS excitation. This research is partly supported by NSF grants AST 87-02945 and RII 86-20342 to Princeton University. 
Adams, N. G., Smith, D., and Clary, D. C. 1985, Ap. J.(Letters), 296, L31. Allison, A. C., and Dalgarno, A. 1971, Astr. Ap., 13, 331

Blitz, L., Magnani, L., and Mundy, L. 1984, Ap. J. (Letters), 282, L9.

Carlson, T. A., Copley, J., Durić, N., Erman, P., and Larsson, M. 1979, Chem. Phys., 42, 81

Chu, S.-I, and Dalgarno, A. 1975, Proc. Roy. Soc. A, 342, 191

Crutcher, R. M. 1985, Ap. J., 288, 604.

Dalgarno, A., and Lepp, S. 1984, Ap. J. (Letters), 287, L47.

de Vries, C. P., and van Dishoeck, E. F. 1988, Astr. Ap., 203, L23.

Dickinson, A. S., Phillips, T. G., Goldsmith, P. F., Percival, I. C., and Richards, D. 1977, Astr. Ap., 54, 645 .

Donovan, R. J., Husain, D., and Stevenson, C. D. 1970, Trans. Faraday Soc., 66, 1 .

Draine, B. T. 1978, Ap. J. Suppl., 36, 595

Duley, W. W., Millar, T. J., and Williams, D. A. 1980, M.N.R.A.S., 192, 945.

Ferlet, R., Roueff, E., Czarny, J., and Felenbok, P. 1986, Astr. Ap., 168, 259.

Ferlet, R., Roueff, E., Horani, M., and Rostas, J. 1983, Astr. Ap., 125, L5.

Gredel, R., and Münch, G. 1986, Astr. Ap., 154, 336.

Green, S., and Chapman, S. 1978, Ap. J. Suppl., 37, 169.

Hartquist, T. W., Flower, D. R., and Pineau des Forêts, G. 1989, in Molecular Astrophysics - A Volume Honoring Alexander Dalgarno, ed. T. W. Hartquist (Cambridge: Cambridge University Press), in press.

Herbst, E., and Leung, C. M. 1986, Ap. J., 310, 378

Huntress, W. T. 1977, Ap. J. Suppl., 33, 495.

Jannuzi, B. T., Black, J. H., Lada, C. J., and van Dishoeck, E. F. 1988, Ap. J., 332, 995 .

Keto, E. R., and Myers, P. C. 1986, Ap. J., 304, 466.

Kirby, K., and Cooper, D. 1989, J. Chem. Phys., 90, 4895.

Knapp, G. R., and Bowers, P. F. 1988, Ap. J., 331, 974

Langer, W. D. Glassgold, A. E., and Wilson, R. W. 1987, Ap. J., 322, 450.

Larsson, M. 1985, Chem. Phys. Letters, 117, 331.

Lepp, S., Dalgarno, A., van Dishoeck, E. F., and Black, J. H. 1988, Ap. J., 329, 418.

Letzelter, C., Eidelsberg, M., Rostas, F., Breton, J., and Thieblemont, B. 1987, Chem. Phys., 114, 273.

Linke, R. A., and Goldsmith, P. F. 1980, Ap. J., 235, 437

Low, F. J., et al. 1984, Ap. J. (Letters), 278, L19.

Marquette, J. B., Rowe, B. R., Dupeyrat, G., Poissant, G., and Rebrion, C. 1985, Chem. Phys. Letters, 122, 431.

Millar, T. J., Adams, N. G., Smith, D., and Clary, D. C. 1985, M.N.R.A.S., 216 , 1025.

K. Drdla: Department of Chemistry, Princeton University, Princeton, NJ 08544.

G. R. KNAPP: Princeton University Observatory, Peyton Hall, Princeton, NJ 08544

E. F. VAN Dishoeck: Division of Geological and Planetary Sciences, California Institute of Technology 170-25, Pasadena, CA 91125
Millar, T. J., Adams, N. G., Smith, D., Lindinger, W., and Villinger, H. 1986, M.N.R.A.S. 221, 673.

Millar, T. J and Hobbs, L. 1988, M.N.R AS, 231, 953.

Morton, D. C. $1975, A p . J ., 197,85$.

Oppenheimer, M., and Dalgarno, A. 1974, Ap. J., 187, 231.

Pes Forêts, G., Flower, D. R., Hartquist, T. W., and Dalgarno, A. 1986 , M.N.R.A.S. 220, 801.

Pineau des Forêts, G., Roueff, E., and Flower, D. R. 1986a, M.N.R.A.S., 223, 743.

Prasad, S. S., and Huntress, W. T. 1980, Ap. J. Suppl., 43, 1.

Rowe.1982, Ap.J., 260, 590.

A. Williams (Dordrecht: Kluwer), p. 135

Sakimoto, K., and Takayanagi, K. 1980, J. Phys. Soc. Japan, 48, 2076.

Snow, T. P. 1975, Ap. J., 201, L21.

1976a, Ap. J. (Letters), 204, L127.

Ap. J. 204,759.

Souza, S. P., and Lutz, B. L. 1977 , Ap. J. (Letters), 216, L49.

Stark, G., Yoshino, K., and Smith, P. L. 1987, J. Molec. Spectrosc., 124, 420.

Su, T., and Bowers, M. T. 1975, in Interactions between Ions and Molecules, ed. P. Ausloos (New York: Plenum).

van Dishoeck, E. F. 1988, in Rate Coefficients in Astrochemistry, ed. T. J. Millar and D. A. Williams (Dordrecht: Kluwer), p. 49. press.

van Dishoeck, E. F., and Black, J. H. 1986, Ap. J. Suppl., 62, 109 (vDB). 1988a, in Rate Coefficients in Astrochemistry, ed. T. J. Millar and D. A. Williams (Dordrecht: Kluwer), p. 209.

1988b, Ap. J., 334, 771

1989, Ap. J. $340,273$.

van Dishoeck, E. F., et al. 1989, in preparation

Viala, Y. P., Letzelter, C., Eidelsberg, M., and Rostas, F. 1988, Astr. Ap., 193, 265.

Winnewisser, G., and Cook, R. L. 1968, J. Molec. Spectrosc., 28, 266

Yoshino, K., Stark, G., Smith, P. L., and Parkinson, W. H. 1989, in preparation.
. 1989, in Molecular Astrophysics - A Volume Honoring Alexander Dal- 